\title{
, Full-Diversity Dispersion Matrices From Algebraic Field Extensions for Differential Spatial Modulation
} \\ Rakshith Rajashekar, Member, IEEE, Naoki Ishikawa, Student Member, IEEE, \\ Shinya Sugiura, Senior Member, IEEE, K. V. S. Hari, Fellow, IEEE, and Lajos Hanzo, Fellow, IEEE
}

\begin{abstract}
5 Abstract-We consider differential spatial modulation (DSM) 6 operating in a block fading environment and propose sparse uni7 tary dispersion matrices (DMs) using algebraic field extensions. 8 The proposed DM sets are capable of exploiting full transmit 9 diversity and, in contrast to the existing schemes, can be con10 structed for systems having an arbitrary number of transmit 11 antennas. More specifically, two schemes are proposed: 1) field12 extension-based DSM (FE-DSM), where only a single conventional 13 symbol is transmitted per space-time block; and 2) FE-DSM 14 striking a diversity-rate tradeoff (FE-DSM-DR), where multiple 15 symbols are transmitted in each space-time block at the cost 16 of a reduced transmit diversity gain. Furthermore, the FE-DSM 17 scheme is analytically shown to achieve full transmit diversity, and 18 both proposed schemes are shown to impose decoding complexity, 19 which is independent of the size of the signal set. It is observed 20 from our simulation results that the proposed FE-DSM scheme 21 suffers no performance loss compared with the existing DM-based 22 DSM (DM-DSM) scheme, whereas FE-DSM-DR is observed to 23 give a better bit-error-ratio performance at higher data rates than 24 its DM-DSM counterpart. Specifically, at data rates of 2.25 and 252.75 bits per channel use, FE-DSM-DR is observed to achieve 26 about 1- and 2-dB signal-to-noise ratio (SNR) gain with respect 27 to its DM-DSM counterpart.
\end{abstract}

28 Index Terms-Decoding complexity, differential spatial modula29 tion (DSM), dispersion matrices (DMs), diversity, field extension.

\section{INTRODUCTION}

32 T $\mathrm{T}$ is widely recognized that multiple-input multiple-output 33 (MIMO) communication systems provide significant spec34 tral efficiency improvements compared with single-input35 single-output systems, owing to their higher degrees of freedom

Manuscript received September 29, 2015; revised December 25, 2015; accepted February 25, 2016. This work was supported in part by the Engineering and Physical Sciences Research Council through projects EP/Noo4558/1 and EP/L018659/1 and in part by the European Research Council's Advanced Fellow Grant through the Beam-Me-Up project and the Royal Society's Wolfson Research Merit Award. The work of N. Ishikawa was supported by the SCAT Fellowship and the MEXT/JASSO Tobitate Fellowship of Japan. The work of S. Sugiura was supported by the Japan Society for the Promotion of Science KAKENHI under Grant 26709028. The review of this paper was coordinated by Prof. H.-F. Lu.

R. Rajashekar and L. Hanzo are with the School of Electronics and Computer Science, University of Southampton, Southampton SO17 1BJ, U.K. (e-mail: rmr1u14@soton.ac.uk; lh@ecs.soton.ac.uk).

N. Ishikawa and S. Sugiura are with the Department of Computer and Information Sciences, Tokyo University of Agriculture and Technology, Koganei 184-8588, Japan (e-mail: sugiura@ieee.org).

K. V. S. Hari is with the Department of Electrical Communication Engineering, Indian Institute of Science, Bangalore 560 012, India (e-mail: hari@ece. iisc.ernet.in).

Color versions of one or more of the figures in this paper are available online at http://ieeexplore.ieee.org.

Digital Object Identifier 10.1109/TVT.2016.2536802
[1]. However, the benefit of increased spectral efficiency comes 36 at the cost of high decoding complexity at the receiver, since 37 the transmitted symbols interfere with each other at the receiver 38 due to the simultaneous activation of multiple transmit antennas 39 (TAs). For instance, in the classic Vertical Bell Laboratories 40 Layered Space-Time architecture [2], the decoding complex- 41 ity of the maximum-likelihood (ML) receiver exponentially 42 increases with the number of TAs. An additional overhead in 43 MIMO systems is that of estimating the channel coefficients 44 between each TA and receive antenna (RA) pair and tracking 45 their changes over the entire transmission duration for coherent 46 detection [4]. Spatial modulation (SM) [5]-[8] is a beneficial 47 multiantenna scheme that overcomes some of these drawbacks. 48 Unlike the conventional MIMO system, the SM system acti- 49 vates only a single TA in each symbol duration, thereby avoid- 50 ing the interference of transmitted symbols with each other at 51 the receiver. As a further substantial benefit, it only requires a 52 single radio frequency (RF) chain, as opposed to $N_{t}$ chains, 53 albeit this potentially precludes having a transmit diversity 54 gain. More specifically, the bitstream is divided into blocks of 55 $\log _{2}\left(M N_{t}\right)$ bits, and in each block, $\log _{2}(M)$ bits are used to 56 select a symbol from an $M$-ary alphabet to be transmitted from 57 a TA chosen from $N_{t}$ TAs based on $\log _{2}\left(N_{t}\right)$ bits. $\quad 58$

The SM system has been extensively studied with regard to 59 various system parameters, which include its transmit diversity 60 order [9]-[12], low-complexity near-ML detection [13]-[17], 61 TA subset selection for performance versus complexity en- 62 hancement [18]-[22], and the impact of channel estimation 63 error on the attainable performance [23]-[25]. A significant 64 research effort was spent on increasing the transmit diversity 65 order of the SM system, since achieving transmit diversity 66 gain in the SM system was not straightforward, owing to 67 the constraint of a single RF chain at the transmitter. This 68 problem was partly addressed by conceiving space-time-coded 69 SM schemes [9]-[12], which operate in an open-loop scenario, 70 and by employing TA subset selection [20], [21], which operate 71 in a closed-loop scenario. Note that both these approaches 72 require accurate channel estimation and tracking at the receiver. 73 Furthermore, the SM system has been studied in nonco- 74 herent communication scenarios [26]-[29], where the high- 75 complexity channel estimation and tracking are dispensed with 76 by employing differential encoding of the transmitted symbols. 77 Naturally, this complexity reduction is achieved at 3-dB per- 78 formance loss. This scheme is referred to as differential SM 79 (DSM) throughout this paper. More specifically, Bian et al. 80 in [26] have extended the conventional SM to a noncoherent 81 scenario by obtaining dispersion matrices (DMs) from a set of 82 
TABLE I

COMPARISON OF VARIOUS EXISTING DSM SCHEMES

\begin{tabular}{|l||c|c|c|}
\hline & P-DSM [26] & DM-DSM [27] & CS-DSM [28] \\
\hline \hline $\begin{array}{l}\text { No. of transmit } \\
\text { RF chains required }\end{array}$ & 1 & 1 & 1 \\
\hline $\begin{array}{l}\text { Throughput } \\
\text { (bpcu) }\end{array}$ & $\frac{\log _{2}\left(M^{N_{t}}\right)+\log _{2}\left\lfloor\left(N_{t} !\right)\right\rfloor_{2} p}{N_{t}}$ & $\frac{\log _{2}\left(M^{N_{t} / d}\right)+\log _{2}(Q)}{N_{t}}$ & $\frac{\log _{2}\left(Q^{\prime} Q\right)}{2}$ \\
\hline $\begin{array}{l}\text { Achievable } \\
\text { diversity } \\
\text { order }\end{array}$ & $N_{r}$ & $\begin{array}{c}d N_{r} \\
\left(1 \leq d \leq N_{t}\right)\end{array}$ & $2 N_{r}$ \\
\hline
\end{tabular}

bpcu : bits per channel use

$83\left(N_{t} \times N_{t}\right)$ permutation matrices having only a single nonzero 84 element in every row and column, where each nonzero ele85 ment is drawn from an $M$-ary phase-shift keying (PSK) signal 86 set. This scheme is referred to as permutation-based DSM 87 (P-DSM). In [27], a fixed set of sparse complex-valued DMs 88 is used in conjunction with a set of diagonal matrices, whose 89 elements are drawn from an $M$-ary PSK signal set. In this 90 scheme, a higher transmit diversity order is shown to be achiev91 able, albeit at the cost of a reduced transmission rate. We refer 92 to this scheme as DM-based DSM (DM-DSM). More recently, 93 a DM set construction was specifically proposed for two TAs 94 [28], where a transmit diversity order of 2 is guaranteed to be 95 achieved. This scheme, which employs a cyclic signal structure 96 based on diagonal matrices along with a set of fixed DMs, is 97 referred to as cyclic-signaling-based DSM (CS-DSM) in this 98 paper. Table I compares these schemes, where $Q$ denotes the 99 number of DMs, $Q^{\prime}$ represents the number of diagonal matrices 100 used for signaling [28], $d$ is the transmit diversity order, and $101\lfloor a\rfloor_{2^{p}}$ denotes the largest integer that is a power of 2 and 102 smaller than $a$, where $d$ is assumed to divide $N_{t}$ with a zero 103 remainder.

104 It is clear from Table I that the DM-DSM achieves the same 105 throughput as that of P-DSM for $d=1$ and $Q=\left\lfloor N_{t} !\right\rfloor_{2^{p}}$, 106 but this will not yield any diversity advantage. To achieve 107 the same throughput as that of P-DSM with full diversity, $108 Q$ should be equal to $M^{N_{t}-1}\left\lfloor N_{t} !\right\rfloor_{2^{p}}$. Similarly, CS-DSM is 109 capable of achieving the same throughput as that of P-DSM 110 for $Q^{\prime}=M^{N_{t}}$ and $Q=\log _{2}\left(\left\lfloor N_{t} !\right\rfloor_{2^{p}}\right)$. However, CS-DSM is 111 specifically designed for the $N_{t}=2$ case, where $Q$ has been 112 restricted to 2 [28]. Furthermore, CS-DSM is different from 113 DM-DSM in the sense that only matrices are used for encoding 114 the information bits, which is in contrast to the DM-DSM, 115 where a set of DMs and a conventional signal set are used for 116 encoding the information bits. To the best of our knowledge, 117 there is no systematic method of obtaining the number of DMs 118 required to achieve a desired throughput and transmit diversity 119 order in systems with arbitrary $N_{t}$. Hence, in this paper, we 120 focus on constructing structured DMs for DM-DSM schemes.

121 Against this background, the contributions of this paper are 122 as follows.

1) We propose a systematic method of obtaining the set of DMs for DSM systems for an arbitrary $N_{t}$ by exploiting the related results from algebraic field extensions. More specifically, we show that the companion matrix of an 127 irreducible polynomial over a certain base field will be 128 unitary, when the base field is a cyclotomic field [30], and 129 exploit these unitary companion matrices for constructing 130 DMs to be used in DSM. Additionally, we analytically 131 show that the proposed scheme is capable of achieving 132 full transmit diversity.

2) Furthermore, we generalize the proposed field-extension- 134 based DSM (FE-DSM) scheme to strike a flexible trade- 135 off between attainable diversity and multiplexing gain. 136

3) Finally, we evaluate the decoding complexity of ML 137 detection of the proposed schemes and show that they 138 offer significantly reduced complexity, owing to the DM- 139 based approach of encoding information by exploiting 140 results from [34].

The rest of this paper is organized as follows. Section II 142 provides the system model of DSM. In Section III, the proposed 143 DM set construction, as well as the diversity analysis of the pro- 144 posed scheme, are presented. Specifically, Section III-A gives 145 a brief overview of algebraic field extensions. Section III-B 146 provides the proposed DM construction and our diversity 147 analysis. In Section III-C, we conceive the low-complexity 148 decoding method for the proposed schemes. Section IV 149 provides our simulation results, and Section V concludes 150 this paper.

151

Notations: If $S_{1}$ and $S_{2}$ are two sets, then $S_{3}=S_{1} \times S_{2} 152$ represents the Cartesian product of sets $S_{1}$ and $S_{2}$. Lowercase 153 and uppercase boldface letters represent vectors and matrices, 154 respectively. Furthermore, $\|\cdot\|$ represents the 2-norm of a 155 vector or the Frobenius norm of a matrix. The notations of $(\cdot)^{T} 156$ and $(\cdot)^{H}$ indicate the transpose and Hermitian transpose of a 157 vector/matrix, respectively, whereas $|\cdot|$ represents the cardi- 158 nality of a given set or the magnitude of a complex quantity. 159 Furthermore, $\otimes$ defines the Kronecker product of two matrices. 160 $\mathcal{C N}\left(\mu, \sigma^{2}\right)$ denotes a complex Gaussian random variable with 161 mean $\mu$ and variance $\sigma^{2} . \mathbb{R}$ and $\mathbb{C}$ represent the field of real 162 and complex numbers, respectively. If $F$ is a field, then $F[X] 163$ represents the ring of polynomials in $X$ over $F . \mathbf{A}([a: b],:) 164$ defines a matrix with rows $a, a+1, \ldots, b-1, b$ of $\mathbf{A}$, and 165 $\mathbf{A}(:,[a: b])$ is a matrix with columns $a, a+1, \ldots, b-1, b$ of 166 A. $\mathbf{I}_{n}$ represents an $n \times n$ identity matrix. If $\mathbf{x}$ is an $n$-length 167 vector, then $\operatorname{diag}(\mathbf{x})$ represents an $n \times n$ diagonal matrix whose 168 $(i, i)$ th element is $\mathbf{x}_{i}$. 


\section{Differential Spatial Modulation System}

171 Consider a MIMO system having $N_{r}$ RAs and $N_{t}$ TAs oper172 ating in a Rayleigh flat-fading channel, which is characterized by

$$
\mathbf{Y}_{i}=\sqrt{\rho} \mathbf{H}_{i} \mathbf{X}_{i}+\mathbf{N}_{i}
$$

173 where $\mathbf{Y}_{i} \in \mathbb{C}^{N_{r} \times N_{t}}$ is the received space-time matrix (STM); $174 \mathbf{X}_{i} \in \mathbb{C}^{N_{t} \times N_{t}}$ is the transmitted STM; $\mathbf{N}_{i} \in \mathbb{C}^{N_{r} \times N_{t}}$ and $\mathbf{H}_{i} \in$ $175 \mathbb{C}^{N_{r} \times N_{t}}$ are the noise and channel matrices, respectively, whose 176 entries are from $\mathcal{C N}(0,1)$; and $\rho$ denotes the average signal-to177 noise ratio (SNR) at each RA. The subscript $i$ in all matrices 178 indicates the block index.

\section{A. DSM System}

180 Differential encoding [31], [32] of the transmitted STM is 181 given by

$$
\mathbf{X}_{i}=\mathbf{X}_{i-1} \mathbf{S}_{i}
$$

182 where $\mathbf{S}_{i} \in \mathbb{C}^{N_{t} \times N_{t}}$ is the unitary STM to be transmitted 183 during the symbol period of the $i$ th block. For the transmitted $184 \mathrm{STM} \mathrm{X}_{i}$ to become unitary, it is sufficient to ensure that $\mathbf{X}_{0}$ be 185 unitary. In this paper, we consider $\mathbf{X}_{0}$ to be $\mathbf{I}_{N_{t}}$. Furthermore, 186 each column of $\mathbf{S}_{i}$ is assumed to have only a single nonzero 187 element, since the SM system employs only a single RF chain 188 at the transmitter. Assuming that the channel remains constant 189 over a period of two successive blocks, we have

$$
\mathbf{Y}_{i-1}=\sqrt{\rho} \mathbf{H}_{i} \mathbf{X}_{i-1}+\mathbf{N}_{i-1}
$$

190 and hence, (1) can be written as

$$
\mathbf{Y}_{i}=\mathbf{Y}_{i-1} \mathbf{S}_{i}+\mathbf{N}_{i}-\mathbf{N}_{i-1} \mathbf{S}_{i}
$$

191 Assuming that there is no channel state information at the 192 receiver, the optimal differential receiver [31] is given by

$$
\hat{\mathbf{S}}_{i}=\arg \min _{\mathbf{S} \in \mathcal{S}}\left\|\mathbf{Y}_{i}-\mathbf{Y}_{i-1} \mathbf{S}\right\|^{2}
$$

193 where $\mathcal{S}$ is the set of transmit STMs.

\section{B. DM-DSM}

195 In the case of DM-DSM, each transmitted STM is of the 196 following form:

$$
\mathbf{S}_{i}=\mathbf{D}(\mathbf{s}) \mathbf{A}_{q}
$$

197 where we have $\mathbf{s}=\left[s_{1}, s_{2}, \ldots, s_{N_{t}}\right], \mathbf{D}(\mathbf{s}) \in \mathcal{D}=\left\{\operatorname{diag}(\mathbf{s}) \mid s_{i} \in\right.$ $\left.198 \mathcal{L}_{i}-\mathrm{PSK}\right\}$, and $\mathbf{A}_{q} \in \mathcal{A}$, where $\mathcal{A}=\left[\mathbf{A}_{1}, \mathbf{A}_{2}, \ldots, \mathbf{A}_{Q}\right]$ is the 199 set of DMs. The rate achieved by DM-DSM is given by

$$
R_{\mathrm{DM}-\mathrm{DSM}}=\frac{\log _{2}\left(Q \cdot \mathcal{L}_{1} \cdots \mathcal{L}_{N_{t}}\right)}{N_{t}} \text { bpcu. }
$$

200 In the following section, we propose a method for construct201 ing the set $\mathcal{D}$ having diagonal or block-diagonal matrices as its 202 elements and the set of DMs $\mathcal{A}$, such that they enable the DSM 203 scheme to achieve full transmit diversity.
C1: We emphasize the condition that each element of $\mathcal{A} 204$ should be a unitary matrix [32] and should have only a single 205 nonzero element in each column and row. The latter condition is 206 necessary since the SM system can transmit only one symbol in 207 each channel use, owing to a single RF chain at the transmitter. 208

\section{Dispersion Matrix Set Construction}

Here, we provide a brief overview of algebraic field exten- 210 sions as required for our exposition on the proposed DM set 211 construction. For further details, see [30] and [33].

\section{A. Review of Field Extensions}

Definitions: Let $J$ be an extension of a field $L$ and $I$ be a 214 subset of $J$, i.e., $I \subset J$. Field $J$ is said to be generated by $I 215$ if $J$ is obtained by adjoining ${ }^{1}$ the elements of $I$ to $L$, and it 216 is denoted by $J=L(I)$. If set $I$ is finite, then the extension, 217 which is denoted by $J / L$, is said to be finitely generated. 218 If $\beta \in J$, then the minimal polynomial of $\beta$ is the monic 219 polynomial of least degree among the polynomials in $L[X] 220$ having $\beta$ as a root. The extended field $J$ can be viewed as 221 a vector space, where its elements are considered as vectors, 222 and the elements of $L$ are viewed as scalars. The dimension of 223 the vector space $J$ is termed as the degree of extension, and it 224 is denoted by $[J: L]$. Furthermore, the extension $J / L$ is said 225 to be an algebraic extension, if every element in $J$ is a root 226 of a nonzero polynomial with coefficients in $L$. An algebraic 227 extension $J / L$ is said to be normal if $J$ is a splitting field of 228 the family of polynomials $L[X]$, i.e., each polynomial in $L[X] 229$ splits or decomposes into linear factors over $J$. Furthermore, an 230 algebraic extension $H$ of $J$ is said to be a normal closure of 231 the algebraic extension $J / L$, if it is the only subfield of $H$ that 232 contains $J$ and if a normal extension of $L$ is $H$ itself.

233

Let $S$ be a conventional signal set, such as $M$-PSK, and $F=234$ $\mathbb{Q}(S)$ be the extended field of rationals over $S$. If $\alpha$ is a root of 235 a minimal polynomial over $F$, which is given by

$$
p(x)=x^{n}+a_{n-1} x^{n-1}+a_{n-2} x^{n-2}+\cdots+a_{0}
$$

then $F$ can be extended by adjoining $\alpha$ to obtain $K=F(\alpha) .237$ The degree of extension $[K: F]$ is equal to $n$, since $p(x)$ is 238 irreducible over $F$. Any element $k \in K$ can be expressed as 239 $\sum_{i=0}^{n-1} f_{i} \alpha^{i}$, where $f_{i} \in F \forall 0 \leq i \leq n-1$. From [30, Sec. 7.3], 240 there exists a natural mapping $k \mapsto \lambda_{k} \forall k \in K$ that embeds $K 241$ in $\mathbf{M}_{n}(F)$, where $\lambda_{k}$ is a linear transformation of $K$ into itself. 242 The regular representation of $\lambda_{k}$ maps any $v \in K$ to $k v$. The 243 linear transformation $\lambda_{\alpha}$ associated with $\alpha$ is given by

$$
\mathbf{M}=\left[\begin{array}{ccccc}
0 & 0 & \cdots & 0 & -a_{0} \\
1 & 0 & \ldots & 0 & -a_{1} \\
0 & 1 & \ldots & 0 & -a_{2} \\
\vdots & \vdots & \ddots & \vdots & \vdots \\
0 & 0 & \cdots & 1 & -a_{n-1}
\end{array}\right] \in F^{n \times n}
$$

\footnotetext{
${ }^{1}$ The adjoining operation refers to including all the elements resulting from field operations considering the elements from the extended set $I \cup L$.
} 
245 which is the companion matrix of $p(x)$. Thus, for any $k=$ $246 \sum_{i=0}^{n-1} f_{i} \alpha^{i} \in K$, the associated $\lambda_{k}$ is given by $\sum_{i=0}^{n-1} f_{i} \mathbf{M}^{i}$.

247 Lemma 1: Let $K, F$, and $S$ be defined as above. For any $248 k=k_{1}-k_{2}, k_{1} \neq k_{2} \in K, \lambda_{k} \in \mathbf{M}_{n}(F)$ is invertible.

249 Proof: The proof directly follows from $K$ being a field, 250 which guarantees the existence of the inverse for every nonzero 251 element in $K$, and the fact that the natural mapping $k \mapsto \lambda_{k}$ is 252 a one-to-one mapping.

253 Lemma 2: If $L$ is a normal closure of $K / F$ and $\sigma_{i}, i=$ $2540,1,2, \ldots, n-1$ are distinct $F$-homomorphisms from $K$ to $L$; 255 then, for any element $k \in K$, we have $\operatorname{det}\left(\lambda_{k}\right)=N_{K / F}(k)=$ $256 \prod_{i=0}^{n-1} \sigma_{i}(k)$, where $N_{K / F}(k)$ is the norm of the element $k$ from $257 K$ to $F[33$, Th. 8].

\section{B. Proposed DM Set for DSM}

259 We propose to use the DM set given by

$$
\mathcal{A}=\left\{\mathbf{I}_{n}, \mathbf{M}, \mathbf{M}^{2}, \ldots, \mathbf{M}^{n-1}\right\}
$$

260 where $\mathbf{M}$ is as in (5), and $n$ is chosen to be equal to $N_{t}$. 261 However, to meet $C 1$, every element of $\mathcal{A}$ has to be unitary. 262 Note that it is sufficient to ensure that $M$ is unitary for all 263 the elements of $\mathcal{A}$ to be unitary. Hence, we have to satisfy the 264 following equation:

$$
\mathbf{M M}^{H}=\mathbf{I}_{n} .
$$

265 Note that $\left(\mathbf{M M}^{H}\right)_{1,1}=\left|a_{0}\right|^{2}$ and $\left(\mathbf{M M}^{H}\right)_{i, i}=1+\left|a_{i-1}\right|^{2}$ for $2662 \leq i \leq n-1$. Thus, by choosing $a_{0}$ to be an element from the 267 unit circle and $a_{i}=0$ for $1 \leq i \leq n-1, C 1$ can be satisfied. 268 Thus, while constructing $\mathcal{A}$, we have to consider polynomials of 269 the form $x^{n}+a_{0}$ with $\left|a_{0}\right|=1$ values that are irreducible over $F$. 270 Since we have $|\mathcal{A}|=n=N_{t}$, our construction results in a max271 imum of $N_{t}$ DMs, i.e., $Q \leq N_{t}$. Furthermore, we assume that the 272 set $\mathcal{D}$ has scaled identity matrices of the form $s \mathbf{I}_{n}$, where $s \in S$. 273 Note that $F$ should contain the specific signal set $S$ from which $274 s$ is chosen. Thus, the following conditions have to be met:

276 1) $S \subset F$; and

277 2) $p(x)=x^{n}+a_{0}$ with $\left|a_{0}\right|=1$ should be irreducible over $F$.

278 We satisfy the given conditions by choosing $F=\mathbb{Q}\left(S, a_{0}\right)$, 279 where $a_{0}$ is any transcendental element over $\mathbb{Q}(S)$ lying on the 280 unit circle. In the following, we shall explain the method of 281 constructing set $\mathcal{A}$ in detail.

282 Let $S$ be a conventional $M$-PSK signal set denoted by $283\left\{\omega_{M}^{i}\right\}_{i=0}^{M-1}$, where we have $\omega_{M}=e^{j 2 \pi / M}$ and $a_{0}=-e^{j u_{1}}$, 284 with $u_{1}$ being algebraic over $\mathbb{Q}$. For instance, $u_{1}$ can be $\sqrt{3}$, 285 which is a root of the polynomial $x^{2}-3$. Note that $a_{0}$ is 286 transcendental over $\mathbb{Q}(S)$, and we can choose $F=\mathbb{Q}\left(S, e^{j u_{1}}\right)$. 287 Thus, the polynomial $x^{n}+a_{0}=x^{n}-e^{j u_{1}}$ (for any $n$ ) is ir288 reducible over $F$. Therefore, we can have the extension $K=$ $289 F(\alpha)$, where $\alpha$ is the primitive $n$th root of $e^{j u_{1}}$. Thus, the 290 associated companion matrix is given by

$$
\mathbf{M}=\left[\begin{array}{ccccc}
0 & 0 & \cdots & 0 & e^{j u_{1}} \\
1 & 0 & \cdots & 0 & 0 \\
0 & 1 & \cdots & 0 & 0 \\
\vdots & \vdots & \ddots & \vdots & \vdots \\
0 & 0 & \cdots & 1 & 0
\end{array}\right] \in F^{n \times n} .
$$

Example 1: Consider $n=N_{t}=4$ and $a_{0}=-e^{j \sqrt{3}}$. Then, 291 the elements of set $\mathcal{A}$ are given by $\mathbf{I}_{4}$

$$
\begin{aligned}
\mathbf{M} & =\left[\begin{array}{cccc}
0 & 0 & 0 & e^{j \sqrt{3}} \\
1 & 0 & 0 & 0 \\
0 & 1 & 0 & 0 \\
0 & 0 & 1 & 0
\end{array}\right], \mathbf{M}^{2}=\left[\begin{array}{cccc}
0 & 0 & e^{j \sqrt{3}} & 0 \\
0 & 0 & 0 & e^{j \sqrt{3}} \\
1 & 0 & 0 & 0 \\
0 & 1 & 0 & 0
\end{array}\right] \\
\mathbf{M}^{3} & =\left[\begin{array}{cccc}
0 & e^{j \sqrt{3}} & 0 & 0 \\
0 & 0 & e^{j \sqrt{3}} & 0 \\
0 & 0 & 0 & e^{j \sqrt{3}} \\
1 & 0 & 0 & 0
\end{array}\right] .
\end{aligned}
$$

Remark 1: Note that $a_{0}$ has to be chosen in conjunction with 293 the specific signal set $S$ that maximizes a certain performance 294 metric, such as the coding gain. This can be achieved by 295 searching for an optimal $a_{0}$ over a large set of closely spaced 296 transcendental elements on the unit circle.

297

We term the DSM scheme employing the proposed FE-DMs 298 as an FE-DSM scheme. Since the set of transmit STMs is given 299 by $\mathcal{S}=\mathcal{D} \times \mathcal{A}$, the rate achieved by the proposed scheme is $\quad 300$

$$
\begin{aligned}
R_{\mathrm{FE}-\mathrm{DSM}} & =\frac{\log _{2}(|\mathcal{D} \| \mathcal{A}|)}{N_{t}} \\
& =\frac{\log _{2}\left(M N_{t}\right)}{N_{t}} \text { bpcu. }
\end{aligned}
$$

1) Diversity Gain: The achievable transmit diversity order 301 under differential detection [31, Sec. III-C] of (2) is given by 302

$$
d=\min _{\mathbf{S}_{1} \neq \mathbf{S}_{2} \in \mathcal{S}} \operatorname{rank}\left(\mathbf{S}_{1}-\mathbf{S}_{2}\right) .
$$

Proposition 1: The proposed FE-DSM scheme achieves a 303 transmit diversity order of $N_{t}$, i.e., $d=N_{t}$.

Proof: The proof is given in Appendix A.

2) Coding Gain: The coding gain of the proposed scheme is 306 given by

$$
G=\min _{\mathbf{S}_{1} \neq \mathbf{S}_{2} \in \mathcal{S}}\left|\operatorname{det}\left[\left(\mathbf{S}_{1}-\mathbf{S}_{2}\right)\left(\mathbf{S}_{1}-\mathbf{S}_{2}\right)^{H}\right]\right|^{\frac{1}{n}} .
$$

In the following, we shall provide a simple expression for 308 the determinant term in (10) that allows us to optimize the 309 exponential $a_{0}$ in conjunction with an arbitrary $M$-PSK signal 310 set to achieve a high coding gain.

Proposition 2: Consider an FE-DSM system using an $M$-PSK 312 signal set and $N_{t}=n$ TAs. If $\mathbf{S}=e^{j(2 \pi p / M)} \mathbf{M}^{l}$ and $\mathbf{S}_{2}=313$ $e^{j(2 \pi q / M)} \mathbf{M}^{m}$, where $0 \leq p, q \leq M-1$ and $0 \leq l, m \leq n-1314$ such that $\mathbf{S}_{1} \neq \mathbf{S}_{2}$, then $\left|\operatorname{det}\left[\left(\mathbf{S}_{1}-\mathbf{S}_{2}\right)\left(\mathbf{S}_{1}-\mathbf{S}_{2}\right)^{H}\right]\right|$ is given by 315

$$
4^{n} \prod_{r=0}^{n-1} \sin ^{2}\left(\frac{\pi(p-q)}{M}+\frac{\left(2 \pi r+u_{1}\right)(m-l)}{2 n}\right) .
$$

Proof: The proof is provided in Appendix B.

In the following section, we provide a DM set construction 317 based on two levels of field extensions, which facilitate a 318 flexible tradeoff between the attainable transmit diversity and 319 multiplexing gain. 


\section{C. FE-DSM With Diversity-Rate Tradeoff}

322 The DM set construction presented in the previous section 323 achieves a transmit diversity order of $N_{t}$, while transmitting 324 only a single symbol from an $M$-PSK signal set. Note that when 325 the channel conditions are good, it may not be necessary to 326 exploit the full transmit diversity order. Under these conditions, 327 we may aim at trading off the diversity gain for increasing 328 the transmission rate. In the following, we shall provide a 329 systematic method of constructing a DM set that achieves 330 the desired diversity order and transmission rate. The DM set 331 construction presented in the previous section may be viewed 332 as a special case.

333 Let $N_{t}$ be factored as $g \cdot h$. We construct a DM set that allows 334 us to transmit $h$ independent $M$-PSK symbols in each transmit 335 STM and achieve transmit diversity order $g$. Considering $F=$ $336 \mathbb{Q}\left(S,-e^{j u_{1}}\right)$ as before and the extension $K=F(\alpha)$, where $\alpha$ 337 is a primitive $g$ th root of the polynomial $p_{1}(x)=x^{g}-e^{j u_{1}}$, we 338 obtain the DM set given by

$$
\mathcal{A}^{\prime}=\left\{\mathbf{I}_{g}, \mathbf{M}, \mathbf{M}^{2}, \ldots, \mathbf{M}^{g-1}\right\}
$$

339 where $\mathbf{M} \in F^{g \times g}$ is the companion matrix of $p_{1}(x)$. We define $340 \mathcal{D}$ to be a set of block-diagonal matrices given by

$$
\mathcal{D}=\left\{\operatorname{diag}\left(s_{1} \mathbf{A}_{1}, s_{2} \mathbf{A}_{2}, \ldots, s_{h} \mathbf{A}_{h}\right) \mid s_{i} \in M-\mathrm{PSK}, \mathbf{A}_{i} \in \mathcal{A}^{\prime}, \forall i\right\} .
$$

341 Let us now consider the field extension $L=K(\beta)$ associated 342 with the polynomial $p_{2}(x)=x^{h}-e^{j u_{2}}$, where $e^{j u_{2}}$ is tran343 scendental over $K$, and $\beta$ is the primitive $h$ th root of $e^{j u_{2}}$. Then, 344 the regular representation of an element $l=\sum_{i=0}^{h-1} k_{i} \beta^{i} \in L$ 345 is given by $\sum_{i=0}^{h-1} k_{i} \mathbf{N}^{i}$, where $k_{i} \in K, 0 \leq i \leq h-1$, and $346 \mathbf{N} \in K^{h \times h}$ is the companion matrix of $p_{2}(x)$. We define the $347 \mathrm{DM}$ set as

$$
\mathcal{A}=\left\{\mathbf{I}_{n}, \mathbf{N}^{\prime}, \mathbf{N}^{\prime 2}, \ldots, \mathbf{N}^{\prime h-1}\right\}
$$

348 where $\mathbf{N}^{\prime}=\mathbf{N} \otimes \mathbf{I}_{g}$. The transmit STM set is given by $349 \mathcal{S}=\mathcal{D} \times \mathcal{A}$ as before. We refer to this scheme as the FE-DSM 350 arrangement exhibiting a flexible diversity-rate tradeoff (FE351 DSM-DR). Note that the DSM scheme requires each transmit 352 STM to be unitary. The following proposition shows that this 353 condition is satisfied.

354 Proposition 3: If $\mathcal{S}$ is the set of transmit STMs of FE-DSM$355 \mathrm{DR}$, then each element in $\mathcal{S}$ is unitary.

356 Proof: The proof is provided in Appendix C.

357 In the following, we shall provide an example construction 358 to further illustrate the given set of points.

359 Since we have $|\mathcal{D}|=(M g)^{h}$ and $|\mathcal{A}|=h$, the rate achieved 360 by the FE-DSM-DR is given by

$$
R_{\mathrm{FE}-\mathrm{DSM}-\mathrm{DR}}=\frac{h \log _{2}(M g)+\log _{2}(h)}{N_{t}} \text { bpcu. }
$$

361 Note that when we have $g=N_{t}$, FE-DSM-DR reduces to the 362 FE-DSM scheme.
Example 2: Let $n=N_{t}=4, g=h=2, u_{1}=\sqrt{2}$, and 363 $u_{2}=\sqrt{3}$. The elements of set $\mathcal{D}$ are

364

$$
\begin{aligned}
& {\left[\begin{array}{cccc}
s_{1} & 0 & 0 & 0 \\
0 & s_{1} & 0 & 0 \\
0 & 0 & s_{2} & 0 \\
0 & 0 & 0 & s_{2}
\end{array}\right],\left[\begin{array}{cccc}
0 & s_{1} e^{j \sqrt{2}} & 0 & 0 \\
s_{1} & 0 & 0 & 0 \\
0 & 0 & s_{2} & 0 \\
0 & 0 & 0 & s_{2}
\end{array}\right]} \\
& {\left[\begin{array}{cccc}
0 & s_{1} e^{j \sqrt{2}} & 0 & 0 \\
s_{1} & 0 & 0 & 0 \\
0 & 0 & 0 & s_{2} e^{j \sqrt{2}} \\
0 & 0 & s_{2} & 0
\end{array}\right],\left[\begin{array}{cccc}
s_{1} & 0 & 0 & 0 \\
0 & s_{1} & 0 & 0 \\
0 & 0 & 0 & s_{2} e^{j \sqrt{2}} \\
0 & 0 & s_{2} & 0
\end{array}\right]}
\end{aligned}
$$

where $s_{1}$ and $s_{2}$ are from the classic $M$-PSK signal set. The 365 elements of the DM set $\mathcal{A}$ are

366

$$
\left[\begin{array}{llll}
1 & 0 & 0 & 0 \\
0 & 1 & 0 & 0 \\
0 & 0 & 1 & 0 \\
0 & 0 & 0 & 1
\end{array}\right],\left[\begin{array}{cccc}
0 & 0 & e^{j \sqrt{3}} & 0 \\
0 & 0 & 0 & e^{j \sqrt{3}} \\
1 & 0 & 0 & 0 \\
0 & 1 & 0 & 0
\end{array}\right]
$$

Remark 2: Note that $e^{j u_{1}}$ and $e^{j u_{2}}$ have to be optimized 367 in conjunction with the signal set $S$ to maximize the coding 368 gain. Unlike FE-DSM, the STM matrices of the FE-DSM-DR 369 scheme are not representations of field elements, and hence, no 370 closed-form expression is derived for the determinant of the 371 codeword difference matrix. We resort to numerical search to 372 arrive at the optimal values of $u_{1}$ and $u_{2}$.

373

\section{ML Decoding Complexity}

Here, we evaluate the complexity order of ML decoding 375 for the proposed schemes. We show that the ML decoding 376 complexity of both proposed schemes is independent of the size 377 of the signal set $S$.

1) FE-DSM: Let $\chi=\left\{s \mathbf{e}_{i} \mid 1 \leq i \leq Q, s \in S\right\}$, where $\mathbf{e}_{i}$ is 379 the $i$ th column of $\mathbf{I}_{Q}$. Furthermore, let $\mathbf{G}=\left[\operatorname{vec}\left(\mathbf{A}_{1}\right)\right.$, $\operatorname{vec}\left(\mathbf{A}_{2}\right), 380$ $\left.\ldots, \operatorname{vec}\left(\mathbf{A}_{Q}\right)\right] \in \mathbb{C}^{N_{t}^{2} \times Q}$, where $\mathbf{A}_{i}$ values are the elements of $\mathcal{A} .381$ Considering the optimal detection rule of (2), we have

$$
\begin{aligned}
\hat{\mathbf{S}}_{i} & =\arg \min _{\mathbf{S} \in \mathcal{S}}\left\|\mathbf{Y}_{i}-\mathbf{Y}_{i-1} \mathbf{S}\right\|^{2} \\
& \equiv \arg \min _{s \in S, \mathbf{A}_{q} \in \mathcal{A}}\left\|\mathbf{Y}_{i}-\mathbf{Y}_{i-1}\left(s \mathbf{A}_{q}\right)\right\|^{2} \\
& \equiv \arg \min _{\mathbf{s} \in \chi}\left\|\overline{\mathbf{Y}}_{i}-\left(\mathbf{I}_{N_{t}} \otimes \mathbf{Y}_{i-1}\right) \mathbf{G s}\right\|^{2}
\end{aligned}
$$

where $\overline{\mathbf{Y}}_{i}=\operatorname{vec}\left(\mathbf{Y}_{i}\right) \in \mathbb{C}^{N_{r} N_{t} \times 1}$. Since we have $|\chi|=Q|S|, 383$ the decoding complexity order is $\mathcal{O}(Q M)$, when $S$ is an 384 $M$-PSK signal set. However, owing to the interference-free 385 nature of transmit vectors, the decoding complexity can be 386 reduced from $\mathcal{O}(Q M)$ to $\mathcal{O}(Q)$ with the aid of hard-limiting 387 (HL)-based detection [34]. In other words, the ML decoding 388 complexity of the FE-DSM scheme does not scale with the size 389 of the signal set. By contrast, the existing full-diversity DSM 390 scheme in [28] does not allow such low decoding complexity. 391 
2) FE-DSM-DR: The optimal detection rule of (2) yields

$$
\begin{aligned}
\hat{\mathbf{S}}_{i} & =\arg \min _{\mathbf{S} \in \mathcal{S}}\left\|\mathbf{Y}_{i}-\mathbf{Y}_{i-1} \mathbf{S}\right\|^{2} \\
& \equiv \arg \min _{\mathbf{D} \in \mathcal{D}, \mathbf{A}_{q} \in \mathcal{A}}\left\|\mathbf{Y}_{i}-\mathbf{Y}_{i-1} \mathbf{D} \mathbf{A}_{q}\right\|^{2} \\
& \equiv \arg \min _{0 \leq k \leq h-1}\left\{\min _{\mathbf{D} \in \mathcal{D}}\left\|\mathbf{Y}_{i}-\mathbf{Y}_{i-1} \mathbf{D N}^{\prime k}\right\|^{2}\right\} \\
\left(\hat{k}, \hat{\mathbf{D}}^{(\hat{k})}\right) & \equiv \arg \min _{0 \leq k \leq h-1}\left\|\mathbf{Z}_{i}^{(k)}-\mathbf{Y}_{i-1} \hat{\mathbf{D}}^{(k)}\right\|^{2}
\end{aligned}
$$

393 where $\hat{\mathbf{D}}^{(k)}=\min _{\mathbf{D} \in \mathcal{D}}\left\|\mathbf{Z}_{i}^{(k)}-\mathbf{Y}_{i-1} \mathbf{D}\right\|^{2}$, and $\mathbf{Z}_{i}^{(k)}=\mathbf{Y}_{i}\left(\mathbf{N}^{\prime k}\right)^{H}$ 394 for $0 \leq k \leq h-1$. Since $\mathbf{D}$ is block diagonal, we have

$$
\begin{aligned}
\hat{\mathbf{D}}^{(k)} & =\min _{\mathbf{D} \in \mathcal{D}}\left\|\mathbf{Z}_{i}^{(k)}-\mathbf{Y}_{i-1} \mathbf{D}\right\|^{2} \\
& \equiv \sum_{l=1}^{h} \min _{s_{l} \in S, \mathbf{A}_{i_{l}} \in \mathcal{A}^{\prime}}\left\|\mathbf{Z}_{i}^{(k)}\left(:, \mathcal{I}_{l}\right)-\mathbf{Y}_{i-1}\left(:, \mathcal{I}_{l}\right)\left(s_{l} \mathbf{A}_{i_{l}}\right)\right\|^{2}
\end{aligned}
$$

395 where $\mathcal{I}_{l}=[g(l-1)+1: g l]$. By invoking the HL-based 396 detector in [34], the search complexity of the minimization 397 problem, i.e.,

$$
\min _{s_{l} \in S, \mathbf{A}_{i_{l}} \in \mathcal{A}^{\prime}}\left\|\mathbf{Z}_{i}^{(k)}\left(:, \mathcal{I}_{l}\right)-\mathbf{Y}_{i-1}\left(:, \mathcal{I}_{l}\right)\left(s_{l} \mathbf{A}_{i_{l}}\right)\right\|^{2}
$$

398 can be reduced from $\mathcal{O}\left(|S|\left|\mathcal{A}^{\prime}\right|\right)$ to $\mathcal{O}\left(\left|\mathcal{A}^{\prime}\right|\right)=\mathcal{O}(g)$. Specifi399 cally, this is achieved by converting (25) into an interference400 free system analogous to (18) and then employing the detector 401 in [34]. Thus, the ML decoding complexity order of FE-DSM$402 \mathrm{DR}$ is independent of the size of the signal set, and it is given 403 by $\mathcal{O}\left(\left|\mathcal{A}^{\prime}\right||\mathcal{A}|\right)=\mathcal{O}(g h)=\mathcal{O}\left(N_{t}\right)$.

\section{E. Computational Complexity}

405 Here, we compare the computational complexity of the ML 406 detector of various existing schemes with that of the proposed 407 scheme. Specifically, we show that all the existing schemes 408 essentially impose the same computational complexity when 409 operating at a given rate. However, since the ML decoding 410 complexity order of the proposed schemes does not scale with 411 the signal set, the computational complexity involved in ML 412 decoding remains constant, when the size of the signal set is 413 increased to increase the transmission rate.

414 Considering the ML detection rule of (2), we have

$$
\hat{\mathbf{S}}_{i}=\arg \min _{\mathbf{S} \in \mathcal{S}}\left\|\mathbf{Y}_{i}-\mathbf{Y}_{i-1} \mathbf{S}\right\|^{2}
$$

415 where $\mathcal{S}$ is the set of transmit STMs. The number of real-valued 416 multiplications in evaluating (26) is $6 N_{r} N_{t}|\mathcal{S}|$, where $|\mathcal{S}|$ is the 417 cardinality of the set of transmit STMs. When the transmission 418 rate is fixed, $|\mathcal{S}|$ is essentially the same across all the existing 419 schemes [26]-[28]. The direct evaluation of (26) results in the 420 same computational complexity across all the schemes, since 421 the number of nonzero elements in each $\mathbf{S} \in \mathcal{S}$ is the same 422 in all of them. However, the proposed FE-DSM (DM-DSM 423 [27]) scheme has the property that $\mathcal{S}=S \times \mathcal{A}$, which makes

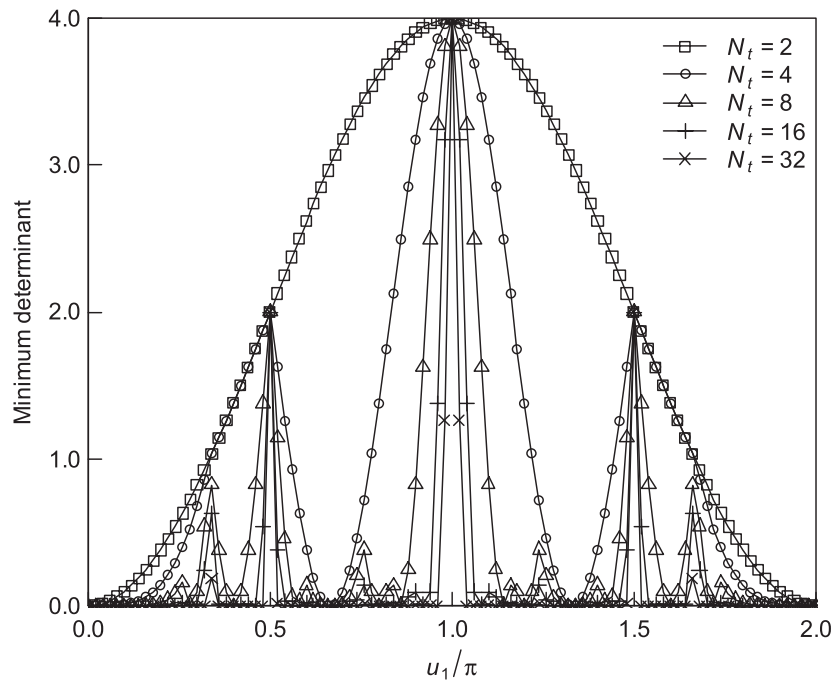

Fig. 1. Variation of coding gain as a function of $u_{1}$ in FE-DSM employing a BPSK signal set for various $N_{t}$ values.

it amenable to HL-based ML detection (HL-ML) [34]. The 424 computational complexity imposed by the HL-ML detector can 425 be shown to be $\left(10 N_{t} N_{r}+9\right)|\mathcal{A}| .^{2}$ In the following section, we 426 compare the computational complexity imposed by the direct 427 ML solution in (26) to that of the HL-ML solution [34] by 428 considering various system parameters and transmission rates. 429

\section{Simulation Results And Discussions}

Simulation Parameters: In all our simulations, we have used 431 block Rayleigh fading channels. In evaluating the bit error ratio 432 (BER) of $10^{-t}$, we have used at least $10^{t+2}$ bits. For DM-DSM 433 schemes operating at different rates, the optimal DM sets are 434 obtained by optimizing the coding gain over a large set of 435 feasible matrices in conjunction with the associated $M$-PSK 436 signal set. The parameter $e^{j u_{1}}$ of FE-DSM and the parameters 437 $\left(e^{j u_{1}}, e^{j u_{2}}\right)$ of FE-DSM-DR are optimized in conjunction with 438 the associated signal sets to obtain the optimal set of DMs. For 439 the FE-DSM scheme using an $M$-PSK signal set, it is observed 440 that $u_{1}=2 \pi / M$ is optimal for any value of $N_{t}$. Fig. 1 shows 441 the achievable coding gain of FE-DSM employing a binary 442 phase-shift keying (BPSK) signal set. It is clear in Fig. 1 that 443 the value of $u_{1}=\pi$ remains optimal even when $N_{t}$ is varied. 444

Fig. 2 compares the BER performance of the FE-DSM and 445 DM-DSM schemes, both having $N_{t}=2$ and employing 4-PSK 446 as well as 16-PSK signal sets that achieve a throughput of 1.5447 and $2.5 \mathrm{bpcu}$, respectively. The BER performance of P-DSM is 448 also provided to highlight the transmit diversity gain achieved 449 by the DM-DSM scheme. Furthermore, the BER performance 450 of the proposed codebooks in the coherent scenario is also pro- 451 vided. Fig. 3 compares the BER performance of the FE-DSM 452 and DM-DSM schemes, both having $N_{t}=4$ and employing 453 4-PSK, as well as 16-PSK signal sets that achieve a throughput 454 of 1 and $1.5 \mathrm{bpcu}$, respectively. It is clear in Figs. 2 and 3 that 455

\footnotetext{
${ }^{2}$ It takes $4 N_{t} N_{r}|\mathcal{A}|$ multiplications to compute $\left(\mathbf{I}_{N_{t}} \otimes \mathbf{Y}_{i-1}\right) \mathbf{G}$ and $\left(6 N_{t} N_{r}+9\right)|\mathcal{A}|$ multiplications to compute various decision metrics of the HL-ML detector [34]. For further details, see [34, Sec. IV-B]
} 


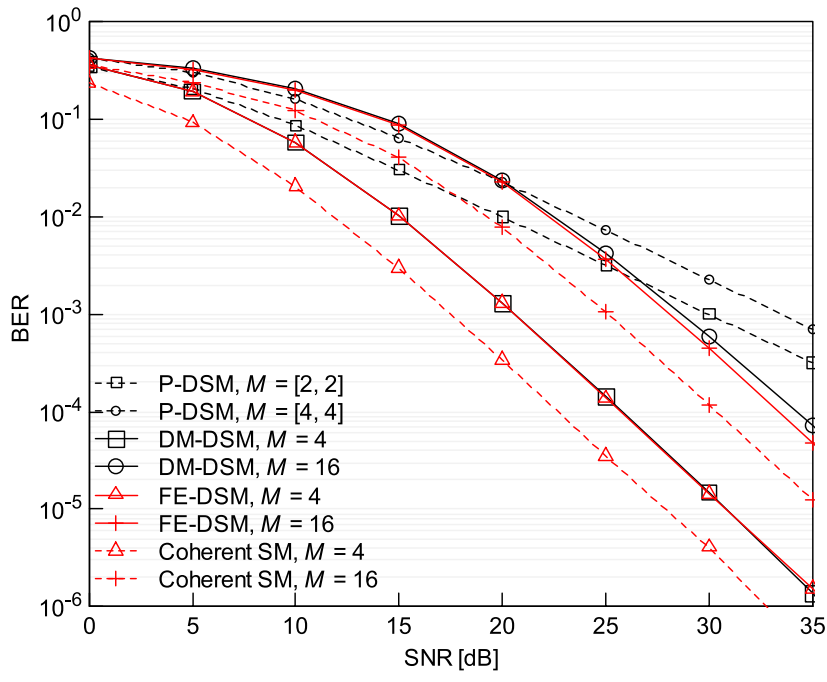

Fig. 2. BER performance of the DM-DSM and FE-DSM schemes, having $N_{t}=2$ and employing 4-PSK and 16-PSK signal sets. The BER performance of the P-DSM scheme is provided to highlight the transmit diversity gain achieved in DM-DSM and FE-DSM.

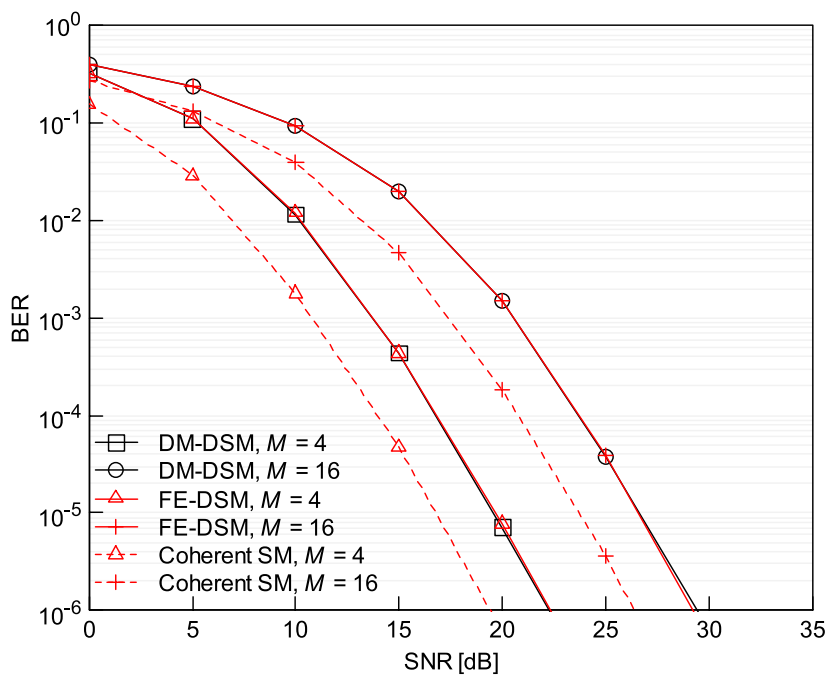

Fig. 3. BER performance of the DM-DSM and FE-DSM schemes, having $N_{t}=4$ and employing $M$-PSK signal sets.

456 the proposed FE-DSM scheme suffers from no performance 457 loss compared with the DM-DSM scheme, and there is a 3$458 \mathrm{~dB}$ performance loss with respect to the coherent counterparts, 459 which is as expected.

460 Fig. 4 compares the BER performance of FE-DSM-DR and 461 DM-DSM that trades off diversity gain against throughput. 462 Both the systems are assumed to have $N_{t}=4$. Specifically, four 463 data rates are considered for comparison. For $h=g=2$, FE464 DSM-DR achieves throughput values of $1.25,1.75,2.25$, and 4652.75 bpcu when employing BPSK, quaternary phase-shift key466 ing, 8-PSK, and 16-PSK signal sets, respectively. The DM467 DSM scheme is assumed to have a set of four DMs as proposed 468 in [27] and employs $M=\left[\left(L_{1}, L_{1}\right),\left(L_{2}, L_{2}\right)\right]$, where $L_{1}$ and $469 L_{2}$ correspond to the sizes of the PSK signal sets encoding sym470 bols $s_{1}$ and $s_{2}$, respectively. To elaborate, $L_{1}$ and $L_{2}$ are chosen 471 so that the rates achieved by the proposed scheme and the 472 FE-DSM scheme are the same. When operating at $1.25 \mathrm{bpcu}$,

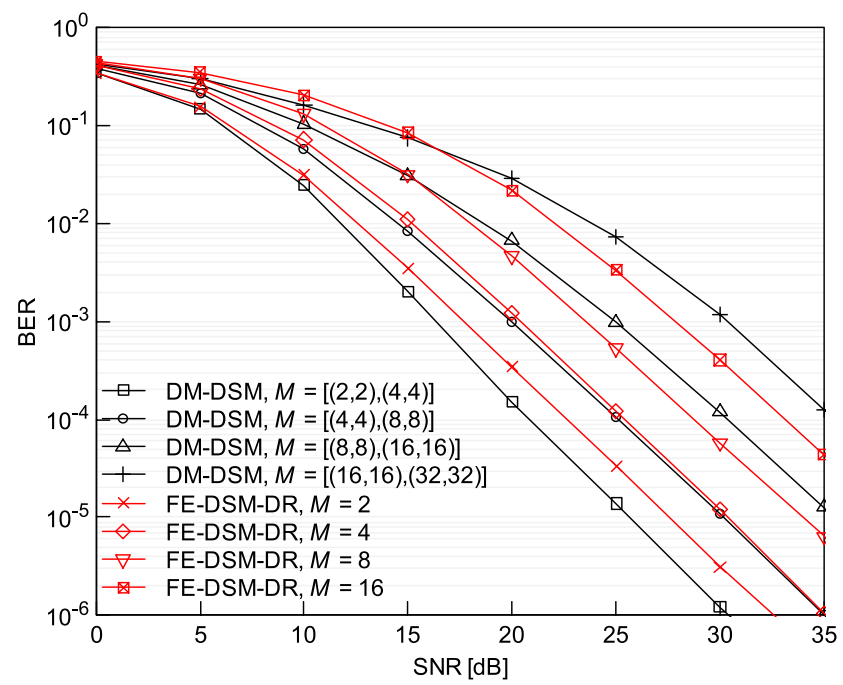

Fig. 4. BER performance of the DM-DSM and FE-DSM-DR schemes, having $N_{t}=4$ and employing $M$-PSK signal sets.

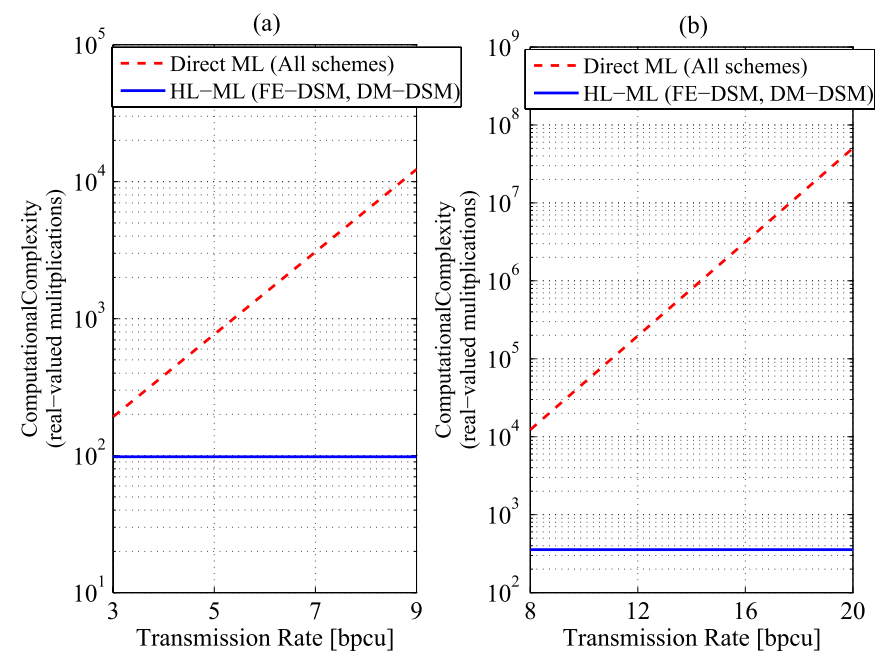

Fig. 5. Comparison of the computational complexity imposed by the ML detector in the existing schemes with that of the proposed scheme in systems having $N_{t}=2,4$ and $N_{r}=2$ and employing various transmission rates.

it can be observed in Fig. 4 that FE-DSM-DR suffers from a 473 1.5-dB SNR loss at a BER of $10^{-4}$ compared with DM-DSM. 474 However, as the rate is increased from 1.25 to $2.75 \mathrm{bpcu}$, the 475 performance of FE-DSM-DR improves, which is evident in 476 Fig. 4. Specifically, when operating at 2.25 and $2.75 \mathrm{bpcu}$, it is 477 observed that the FE-DSM-DR scheme achieves an SNR gain 478 of about $1 \mathrm{~dB}$ and about $2 \mathrm{~dB}$ at a BER of $10^{-4}$, respectively. 479

Fig. 5 gives the computational complexity imposed by the 480 ML detector in various existing schemes along with that of the 481 proposed FE-DSM scheme in systems having $N_{t}=2,4$ and 482 $N_{r}=2$ and employing various transmission rates. In the case 483 of $N_{t}=2$, the P-DSM scheme is assumed to employ BPSK, 484 4-PSK, 8-PSK, and 16-PSK to achieve a transmission rate of 485 $3,5,7$, and $9 \mathrm{bpcu}$, respectively, and in the case of $N_{t}=4,486$ the same signal sets achieve a transmission rate of $8,12,16,487$ and $20 \mathrm{bpcu}$, respectively. In the case of FE-DSM, the size 488 of the DM set is fixed to 2 and 4 in the case of $N_{t}=2$ and 489 $N_{t}=4$, respectively, and the size of the signal set (or the 490 
491 number of cyclic matrices in the case of CS-DSM [26] when $492 N_{t}=2$ ) is assumed to vary to increase the transmission rate. It 493 is clear in Fig. 5 that the HL-ML detector results in significant 494 complexity reductions over the direct ML solution. Specifically, 495 at a transmission rate of 5 and $8 \mathrm{bpcu}$, a reduction of about 496670 multiplications in the case of $N_{t}=2$ and about 11934 497 multiplications in the case of $N_{t}=4$ is observed, respectively. 498 Future Work: While the proposed DM set constructions cater 499 to the requirements of the SM scheme relying on differential 500 encoding, it would be interesting to study the feasibility of ex501 tending the proposed schemes to generalized SM (GSM) [35], 502 where more than one TAs are activated during each channel use. 503 Note, however, that this is not straightforward, since

504

505 1) the transmitted STMs in GSM may not be unitary in 506 general; and

507 2) the product of any two distinct transmit STMs does not 508 satisfy the sparsity constraint analogous to condition C1 509 given in Section II-B.

510 As inferred from Fig. 4, the FE-DSM-DR-based DMs are not 511 optimal at low rates (unlike the FE-DSM scheme). Furthermore, 512 considering algebraic structures for designing DM sets would 513 enable us to quantify the achievable diversity order and the cod514 ing gain in addition to attaining the benefits of a simple and sys515 tematic encoding at the transmitter. Thus, worth investigating 516 are other representations of algebraic structures such as division 517 algebras for their suitability in obtaining sparse, full-diversity, 518 and optimal DM sets for differential SM/GSM schemes.

\section{CONCLUSION}

520 We have proposed a systematic method for obtaining a DM 521 set for DSM with the aid of algebraic field extensions. It was 522 analytically shown that the proposed FE-DSM achieves full 523 transmit diversity. Furthermore, a closed-form expression was 524 derived for the determinant of the codeword difference matrix. 525 The proposed FE-DSM scheme was then further extended to 526 FE-DSM-DR, which stroke a flexible tradeoff between diver527 sity gain and throughput. Both the proposed schemes were 528 shown to offer ML decoding complexity, which is independent 529 of the size of the signal set. Our simulation results have shown 530 that the FE-DSM scheme achieves the same BER performance 531 as the DM-DSM scheme, whereas FE-DSM-DR is observed to 532 give a better BER performance at higher rates compared with 533 its DM-DSM counterpart.

\section{ProOF OF PROPOSITION 1}

536 Let $\mathbf{S}_{1}=s \mathbf{M}^{i}$ and $\mathbf{S}_{2}=s^{\prime} \mathbf{M}^{j}$, where $s, s^{\prime} \in S \subset F=$ $537 \mathbb{Q}\left(S, a_{0}\right)$. Recall that $\mathbf{S}_{1}$ and $\mathbf{S}_{2}$ are regular representations 538 of $k_{1}=s \alpha^{i}$ and $k_{2}=s^{\prime} \alpha^{j}$, respectively. Then, we have $\lambda_{k}=$ $539 \mathbf{S}_{1}-\mathbf{S}_{2}$, where $k=k_{1}-k_{2}$. From Lemma 1 , we see that $\lambda_{k}$ is 540 invertible for all $k_{1}, k_{2} \in K$ and $k_{1} \neq k_{2}$. Thus, we have $\mathbf{S}_{1}-$ $541 \mathbf{S}_{2}$ as invertible. In other words, we have $\operatorname{rank}\left(\mathbf{S}_{1}-\mathbf{S}_{2}\right)=n=$ $542 N_{t}, \forall \mathbf{S}_{1} \neq \mathbf{S}_{2} \in \mathcal{S}$. Thus, we have $\min _{\mathbf{S}_{1} \neq \mathbf{S}_{2} \in \mathcal{S}} \operatorname{rank}\left(\mathbf{S}_{1}-\right.$ $\left.543 \mathbf{S}_{2}\right)=N_{t}$. This concludes the proof.
APPENDIX B

PROOF OF PROPOSITION 2

If $\lambda_{k}=\mathbf{S}_{1}-\mathbf{S}_{2}$, then the element $k$ associated with $\lambda_{k}$ is given 546 by $s \alpha^{m}-s^{\prime} \alpha^{l}$, where $s=e^{j(2 \pi p / M)}, s^{\prime}=e^{j(2 \pi q / M)}$, and $\alpha$ is the 547 primitive $n$th root of $-a_{0}$. From Lemma 2, we have $\operatorname{det}\left(\lambda_{k}\right)=548$ $\operatorname{det}\left(\mathbf{S}_{1}-\mathbf{S}_{2}\right)=\prod_{r=0}^{n-1} \sigma_{r}(k)$, where we have $\sigma_{r}: \alpha \mapsto \alpha_{r}, 549$ such that $\alpha_{r}, 0 \leq r \leq n-1$ are the $n$th roots of $-a_{0}=e^{j u_{1}} .550$ Therefore, we have

$$
\begin{aligned}
\operatorname{det}\left(\mathbf{S}_{1}-\mathbf{S}_{2}\right) & =\prod_{r=0}^{n-1} \sigma_{r}(k)=\prod_{r=0}^{n-1} \sigma_{r}\left(s \alpha^{m}-s^{\prime} \alpha^{l}\right) \\
& =\prod_{r=0}^{n-1}\left(s\left(\sigma_{r}(\alpha)\right)^{m}-s^{\prime}\left(\sigma_{r}(\alpha)\right)^{l}\right) \\
& =\prod_{r=0}^{n-1}\left(e^{j \frac{2 \pi p}{M}+\frac{\left(2 \pi r+u_{1}\right) m}{n}}-e^{j \frac{2 \pi q}{M}+\frac{\left(2 \pi r+u_{1}\right) l}{n}}\right) .
\end{aligned}
$$

Thus, we have

$$
\begin{aligned}
\operatorname{det} & {\left[\left(\mathbf{S}_{1}-\mathbf{S}_{2}\right)\left(\mathbf{S}_{1}-\mathbf{S}_{2}\right)^{H}\right] } \\
= & \mid \prod_{r=0}^{n-1}\left(e^{j \frac{2 \pi p}{M}+\frac{\left(2 \pi r+u_{1}\right) m}{n}}-\left.e^{\left.j \frac{2 \pi q}{M}+\frac{\left(2 \pi r+u_{1}\right) l}{n}\right)}\right|^{2}\right. \\
= & 4^{n} \prod_{r=0}^{n-1} \sin ^{2}\left(\frac{\pi(p-q)}{M}+\frac{\left(2 \pi r+u_{1}\right)(m-l)}{2 n}\right) .
\end{aligned}
$$

This concludes the proof.

\section{APPENDIX C}

Let $\mathbf{S} \in \mathcal{S}$ such that $\mathbf{S}=\operatorname{diag}\left(s_{1} \mathbf{A}_{1}, s_{2} \mathbf{A}_{2}, \ldots, s_{h} \mathbf{A}_{h}\right) \mathbf{N}^{\prime k}{ }_{556}$ for some $0 \leq k \leq h-1, s_{i} \in M$-PSK signal set, $\mathbf{A}_{i} \in \mathcal{A}^{\prime}$ for 557 $1 \leq i \leq h$. Consider $\mathbf{S S}^{H}=\mathbf{D} \mathbf{N}^{\prime k} \mathbf{N}^{\prime{ }^{H}} \mathbf{D}^{H}$, where $\mathbf{D}=558$ $\operatorname{diag}\left(s_{1} \mathbf{A}_{1}, s_{2} \mathbf{A}_{2}, \ldots, s_{h} \mathbf{A}_{h}\right)$. Since $\mathbf{N}^{\prime k}=\left(\mathbf{N} \otimes \mathbf{I}_{g}\right)^{k}=\mathbf{N}^{k} \otimes \mathbf{I}_{g}, 559$ we have

$$
\begin{aligned}
\mathbf{N}^{\prime k} \mathbf{N}^{\prime k} & =\left(\mathbf{N}^{k} \otimes \mathbf{I}_{g}\right)\left(\mathbf{N}^{k} \otimes \mathbf{I}_{g}\right)^{H} \\
& =\left(\mathbf{N}^{k} \mathbf{N}^{k} \otimes \mathbf{I}_{g}\right) \\
& =\mathbf{I}_{h} \otimes \mathbf{I}_{g}=\mathbf{I}_{n}
\end{aligned}
$$

Thus, we have

$$
\begin{aligned}
\mathbf{S S}^{H} & =\mathbf{D D}^{H} \\
& =\operatorname{diag}\left(\mathbf{A}_{1} \mathbf{A}_{1}^{H}, \mathbf{A}_{2} \mathbf{A}_{2}^{H}, \ldots, \mathbf{A}_{h} \mathbf{A}_{h}^{H}\right) \\
& =\operatorname{diag}\left(\mathbf{I}_{g}, \mathbf{I}_{g}, \ldots, \mathbf{I}_{g}\right)=\mathbf{I}_{n} .
\end{aligned}
$$

This concludes the proof. 


\section{REFERENCES}

1] I. E. Telatar, "Capacity of multi-antenna Gaussian channels," Eur. Trans. Telecommun., vol. 10, no. 6, pp. 585-595, Dec. 1999.

2] P. Wolniansky, G. Foschini, G. Golden, and R. Valenzuela, "V-BLAST: An architecture for realizing very high data rates over the rich-scattering wireless channel," in Proc. Int. Symp. Signals, Syst., Electron., Pisa, Italy, Sep. 1998, pp. 295-300.

3] D. Tse and P. Viswanath, Fundamentals of Wireless Communication. Cambridge, U.K.: Cambridge Univ. Press, 2005.

4] B. Hassibi and B. M. Hochwald, "How much training is needed in multiple-antenna wireless links?" IEEE Trans. Inf. Theory, vol. 49, no. 4, pp. 951-963, Apr. 2003.

[5] R. Mesleh, H. Haas, C. Ahn, and S. Yun, "Spatial modulation-A new low complexity spectral efficiency enhancing technique," in Proc. 1st Int. Conf. Commun. Netw., Beijing, China, Oct. 2006, pp. 1-5.

[6] R. Mesleh, H. Haas, S. Sinanovic, C. Ahn, and S. Yun, "Spatial modulation," IEEE Trans. Veh. Technol., vol. 57, no. 4, pp. 2228-2242, Jul. 2008.

[7] M. Di Renzo, H. Haas, and P. M. Grant, "Spatial modulation for multipleantenna wireless systems-A survey," IEEE Commun. Mag., vol. 49, no. 12, pp. 182-191, Dec. 2011.

8] M. Di Renzo, H. Haas, A. Ghrayeb, S. Sugiura, and L. Hanzo, "Spatial modulation for generalized MIMO: Challenges, opportunities, and implementation," Proc. IEEE, vol. 102, no. 1, pp. 56-103, Jan. 2014

[9] E. Basar, U. Aygolu, E. Panayirci, and H. V. Poor, "Space-time block coding for spatial modulation," IEEE Trans. Commun., vol. 59, no. 3, pp. 823-832, Mar. 2011.

0] M. Di Renzo and H. Haas, "Transmit-diversity for spatial modulation (SM): Towards the design of high-rate spatially-modulated space-time block codes," in Proc. IEEE Int. Conf. Commun., Jun. 2011, pp. 1-6.

11] R. Rajashekar and K. V. S. Hari, "Modulation diversity for spatial modulation using complex interleaved orthogonal design," in Proc. IEEE TENCON, Nov. 2012, pp. 1-6.

2] X. Li and L. Wang, "High rate space-time block coded spatial modulation with cyclic structure," IEEE Commun. Lett., vol. 18, no. 4, pp. 532-535, Apr. 2014

13] J. Jeganathan, A. Ghrayeb, and L. Szczecinski, "Spatial modulation: Optimal detection and performance analysis," IEEE Commun. Lett., vol. 12, no. 8, pp. 545-547, Aug. 2008.

14] S. Sugiura, C. Xu, S. X. Ng, and L. Hanzo, "Reduced-complexity coherent versus non-coherent QAM-aided space-time shift keying," IEEE Trans. Commun., vol. 59, no. 11, pp. 3090-3101, Nov. 2011.

15] J. Wang, S. Jia, and J. Song, "Signal vector based detection scheme for spatial modulation," IEEE Commun. Lett., vol. 16, no. 1, pp. 19-21, Jan. 2012.

16] Q. Tang, Y. Xiao, P. Yang, Q. Yu, and S. Li, “A new low-complexity nearML detection algorithm for spatial modulation," IEEE Wireless Commun. Lett., vol. 2, no. 1, pp. 90-93, Feb. 2013.

17] R. Rajashekar, K. V. S. Hari, and L. Hanzo, "Reduced-complexity ML detection and capacity-optimized training for spatial modulation systems," IEEE Trans. Commun., vol. 62, no. 1, pp. 112-125, Jan. 2014

8] P. Yang, Y. Xiao, Y. Yu, and S. Li, "Adaptive spatial modulation for wireless MIMO transmission systems," IEEE Commun. Lett., vol. 15, no. 6, pp. 602-604, Jun. 2011.

19] P. Yang et al., "Link adaptation for spatial modulation with limited feedback," IEEE Trans. Veh. Technol., vol. 61, no. 8, pp. 3808-3813, Oct. 2012.

20] R. Rajashekar, K. V. S. Hari, and L. Hanzo, "Antenna selection in spatial modulation systems," IEEE Commun. Lett., vol. 17, no. 3, pp. 521-524, Mar. 2013.

21] R. Rajashekar, K. V. S. Hari, and L. Hanzo, "Quantifying the transmit diversity order of Euclidean distance based antenna selection in spatial modulation," IEEE Signal Procss. Lett., vol. 22, no. 9, pp. 1434-1437, Sep. 2015.

22] J. Zheng and J. Chen, "Further complexity reduction for antenna selection in spatial modulation systems," IEEE Commun. Lett., vol. 19, no. 6, pp. 937-940, Jun. 2015.

23] E. Basar, U. Aygolu, E. Panayirci, and H. V. Poor, "Performance of spatial modulation in the presence of channel estimation errors," IEEE Commun. Lett., vol. 16, no. 2, pp. 176-179, Feb. 2012.

24] S. Sugiura and L. Hanzo, "Effects of channel estimation on spatial modulation," IEEE Signal Process. Lett., vol. 19, no. 12, pp. 805-808, Dec. 2012.

25] R. Mesleh, O. S. Badarneh, A. Younis, and H. Haas, "Performance analysis of spatial modulation and space-shift keying with imperfect channel estimation over generalized $\eta-\mu$ fading channels," IEEE Trans. Veh. Technol., vol. 64, no. 1, pp. 88-96, Jan. 2015.
[26] Y. Bian et al., "Differential spatial modulation," IEEE Trans. Veh. 639 Technol., vol. 64, no. 7, pp. 3262-3268, Jul. 2015.

[27] N. Ishikawa and S. Sugiura, "Unified differential spatial modulation," 641 IEEE Wireless Commun. Lett., vol. 3, no. 4, pp. 337-340, Aug. 2014.

[28] W. Zhang, Q. Yin, and H. Deng, "Differential full diversity spatial mod- 643 ulation and its performance analysis with two transmit antennas," IEEE 644 Commun. Lett., vol. 19, no. 4, pp. 677-680, Apr. 2015.

[29] M. Wen, X. Cheng, Y. Bian, and H. V. Poor, "A low-complexity near-ML 646 differential spatial modulation detector," IEEE Signal Process. Lett., 647 vol. 22, no. 11, pp. 1834-1838, Nov. 2015.

[30] N. Jacobson, Basic Algebra I, 2nd ed. New York, NY, USA: Wiley, 649 1985.

[31] B. L. Hughes, "Differential space-time modulation," IEEE Trans. Inf. 651 Theory, vol. 46, no. 7, pp. 2567-2578, Nov. 2000.

[32] B. M. Hochwald and W. Sweldens, "Differential unitary space-time 653 modulation," IEEE Trans. Commun., vol. 48, no. 12, pp. 2041-2052, 654 Dec. 2000

[33] B. A. Sethuraman and B. Sundar Rajan, "Full-diversity, high-rate 656 space-time block codes from division algebras," IEEE Trans. Inf. Theory, 657 vol. 49, no. 10, pp. 2596-2616, Oct. 2003.

658

[34] H. Men and M. Jin, "A low-complexity ML detection algorithm for spatial 659 modulation systems with M PSK constellation," IEEE Commun. Lett., 660 vol. 18, no. 8, pp. 1375-1378, Aug. 2014.

[35] J. Wang, S. Jia, and J. Song, "Generalised spatial modulation system 662 with multiple active transmit antennas and low complexity detection 663 scheme," IEEE Trans. Wireless Commun., vol. 11, no. 4, pp. 1605-1615, 664 Apr. 2012.

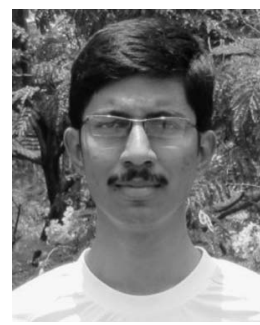

Rakshith Rajashekar (M'15) received the B.E. de- 666 gree in electrical communication engineering from 667 Visvesvaraya Technological University, Belgaum, 668 India, in 2007 and the Ph.D. degree from the 669 Department of Electrical Communication Engineer- 670 ing, Indian Institute of Science, Bangalore, India, 671 in 2014.

672

He is currently a Research Fellow with the Uni- 673 versity of Southampton (UoS), Southampton, U.K. 674 Before joining the UoS, he was a Systems Engineer 675 with Accord Software and Systems, Bangalore, from 676 2007 to 2009 and a Senior Scientist with Broadcom Communications from 677 2014 to 2015. His current research interests include millimeter-wave commu- 678 nication and visible light communication, with a focus on space-time signal 679 processing and coding aspects.

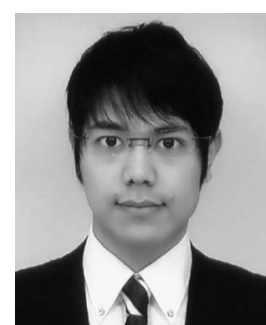

Japanese Government
Naoki Ishikawa (S'13) was born in Kanagawa, 681 Japan, in 1991. He received the B.E. and M.E. 682 degrees (with highest distinction) in computer and 683 information sciences from the Tokyo University of 684 Agriculture and Technology, Koganei, Japan, in 2014685 and 2015, respectively, where he is currently working 686 toward the Ph.D. degree.

687

From June 2015 to September 2015, he was 688 an academic visitor with the School of Electronics 689 and Computer Science, University of Southampton, 690 Southampton, U.K., which is fully funded by the 691

Mr. Ishikawa has received six domestic student awards, including the 693 Telecom System Technology Student Award (honorable mention) from the 694 Telecommunications Advancement Foundation of Japan in 2014, the Outstand- 695 ing Paper Award for Young C\&C Researchers from the NEC Computer and 696 Communications Foundation in 2014, the Young Researcher's Encouragement 697 Award from the IEEE Vehicular Technology Society Japan Chapter in 2014, 698 and three honors from the Tokyo University of Agriculture and Technology. 699 


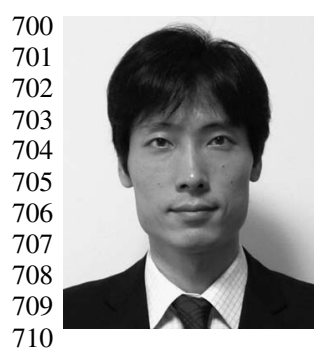

Shinya Sugiura (M'06-SM'12) received the B.S. and M.S. degrees in aeronautics and astronautics from Kyoto University, Kyoto, Japan, in 2002 and 2004, respectively, and the Ph.D. degree in electronics and electrical engineering from the University of Southampton, Southampton, U.K., in 2010.

From 2004 to 2012, he was a Research Scientist with Toyota Central Research and Development Laboratories, Inc., Aichi, Japan. Since 2013, he has been an Associate Professor with the Department of Computer and Information Sciences, Tokyo Uni711 versity of Agriculture and Technology, Koganei, Japan, where he heads the 712 Wireless Communications Research Group. He has authored or coauthored 713 over 70 refereed research publications, including 41 IEEE journal and magazine 714 papers. His research has covered a range of areas in wireless communications, 715 networking, signal processing, and antenna technology.

716 Dr. Sugiura has received a number of awards, including the 14th Funai In717 formation Technology Award (First Prize) from the Funai Foundation in 2015, 718 the 28th Telecom System Technology Award from the Telecommunications 719 Advancement Foundation in 2013, the Sixth IEEE Communications Society 720 Asia-Pacific Outstanding Young Researcher Award in 2011, the 13th Ericsson 721 Young Scientist Award in 2011, and the 2008 IEEE Antennas and Propagation 722 Society Japan Chapter Young Engineer Award. He was also certified as an 723 Exemplary Reviewer for the IEEE COMMUNICATIONS LETTERS in 2013 and 7242014.

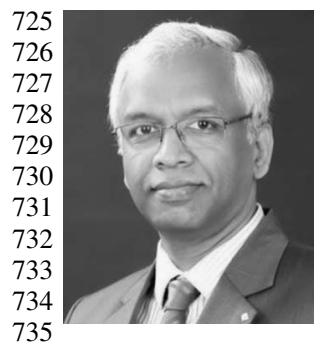

K. V. S. Hari (M'92-SM'97-F'15) received the B.E. degree from Osmania University, Hyderabad, India, in 1983; the M.Tech. degree from the Indian Institute of Technology Delhi, New Delhi, India, in 1985; and the Ph.D. degree from the University of California at San Diego, La Jolla, CA, USA, in 1990.

Since 1992, he has been with the Department of Electrical Communication Engineering, Indian Institute of Science, Bangalore, India, where he is currently a Professor and coordinates the activities of the Statistical Signal Processing Laboratory. He is also 736 an Affiliated Professor with the Department of Signal Processing, KTH Royal 737 Institute of Technology, Stockholm, Sweden. He has been a Visiting Faculty 738 Member with Stanford University, Stanford, CA, USA; KTH Royal Institute 739 of Technology, Stockholm, Sweden; and Aalto University, Espoo, Finland 740 (formerly Helsinki University of Technology). While at Stanford University, he 741 worked on multiple-input multiple-output (MIMO) wireless channel modeling 742 and coauthored the Worldwide Interoperability for Microwave Access stan743 dard on wireless channel models for fixed-broadband wireless communication 744 systems, which proposed the Stanford University Interim channel models. $745 \mathrm{He}$ was also with the Defense Electronics Research Laboratory, Hyderabad, 746 and the Research and Training Unit for Navigational Electronics, Osmania 747 University. His research interests include the development of signal processing 748 algorithms for MIMO wireless communication systems, sparse signal recovery 749 problems, indoor positioning, assistive technologies for the elderly, and visual 750 neuroscience.

751 Dr. Hari is currently an Editor of Elsevier's EURASIP journal Signal 752 Processing and the Senior Associate Editor of Springer's Indian Academy of 753 Sciences journal SADHANA. He received the Institution of Electronics and 754 Telecommunication Engineers S. V. C. Aiya Award for Excellence in Telecom 755 Education and the Distinguished Alumnus Award from the Osmania University 756 College of Engineering, Hyderabad. He is an Academic Entrepreneur and a 757 Cofounder of the company ESQUBE Communication Solutions, Bangalore.

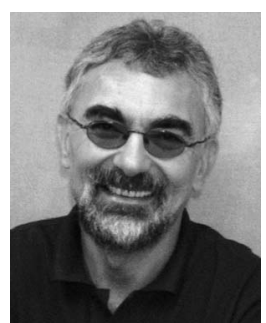

Lajos Hanzo (M'91-SM'92-F'04) received the 758 M.S. degree in electronics and the Ph.D. degree from 759 Budapest University of Technology and Economics 760 (formerly, Technical University of Budapest), 761 Budapest, Hungary, in 1976 and 1983, respec- 762 tively; the D.Sc. degree from the University of 763 Southampton, Southampton, U.K., in 2004; and 764 the "Doctor Honoris Causa" degree from Budapest 765 University of Technology and Economics in 2009. 766

During his 38-year career in telecommunications, 767 he has held various research and academic posts in 768 Hungary, Germany, and the U.K. Since 1986, he has been with the School 769 of Electronics and Computer Science, University of Southampton, where he 770 holds the Chair in Telecommunications. He is currently directing a 100-strong 771 academic research team, working on a range of research projects in the 772 field of wireless multimedia communications sponsored by the industry, the 773 Engineering and Physical Sciences Research Council, the European IST Pro- 774 gram, and the Mobile Virtual Center of Excellence. During 2008-2012, he 775 was a Chaired Professor with Tsinghua University, Beijing, China. He is an 776 enthusiastic supporter of industrial and academic liaison and offers a range of 777 industrial courses. He has successfully supervised more than $80 \mathrm{Ph}$.D. students, 778 coauthored 20 John Wiley/IEEE Press books on mobile radio communications 779 totaling in excess of 10000 pages, and published more than 1300 research 780 entries at IEEE Xplore. He has more than 17000 citations. His research is 781 funded by the European Research Council's Senior Research Fellow Grant. 782 (For further information on research in progress and associated publications, 783 please refer to http://www-mobile.ecs.soton.ac.uk.)

Dr. Hanzo is a Fellow of the Royal Academy of Engineering, The Institution 785 of Engineering and Technology, and the European Association for Signal 786 Processing. He is also a Governor of the IEEE Vehicular Technology Society. 787 He has served as the Technical Program Committee Chair and the General Chair 788 of IEEE conferences, has presented keynote lectures, and has been awarded a 789 number of distinctions. During 2008-2012, he was the Editor-in-Chief of the 790 IEEE Press. 


\section{AUTHOR QUERY}

NO QUERY. 


\title{
, Full-Diversity Dispersion Matrices From Algebraic Field Extensions for Differential Spatial Modulation
}

\author{
Rakshith Rajashekar, Member, IEEE, Naoki Ishikawa, Student Member, IEEE, \\ Shinya Sugiura, Senior Member, IEEE, K. V. S. Hari, Fellow, IEEE, and Lajos Hanzo, Fellow, IEEE
}

\begin{abstract}
5 Abstract-We consider differential spatial modulation (DSM) 6 operating in a block fading environment and propose sparse uni7 tary dispersion matrices (DMs) using algebraic field extensions. 8 The proposed DM sets are capable of exploiting full transmit 9 diversity and, in contrast to the existing schemes, can be con10 structed for systems having an arbitrary number of transmit 11 antennas. More specifically, two schemes are proposed: 1) field12 extension-based DSM (FE-DSM), where only a single conventional 13 symbol is transmitted per space-time block; and 2) FE-DSM 14 striking a diversity-rate tradeoff (FE-DSM-DR), where multiple 15 symbols are transmitted in each space-time block at the cost 16 of a reduced transmit diversity gain. Furthermore, the FE-DSM 17 scheme is analytically shown to achieve full transmit diversity, and 18 both proposed schemes are shown to impose decoding complexity, 19 which is independent of the size of the signal set. It is observed 20 from our simulation results that the proposed FE-DSM scheme 21 suffers no performance loss compared with the existing DM-based 22 DSM (DM-DSM) scheme, whereas FE-DSM-DR is observed to 23 give a better bit-error-ratio performance at higher data rates than 24 its DM-DSM counterpart. Specifically, at data rates of 2.25 and 252.75 bits per channel use, FE-DSM-DR is observed to achieve 26 about 1- and 2-dB signal-to-noise ratio (SNR) gain with respect 27 to its DM-DSM counterpart.
\end{abstract}

28 Index Terms-Decoding complexity, differential spatial modula29 tion (DSM), dispersion matrices (DMs), diversity, field extension.

\section{0 \\ I. INTRODUCTION}

32 T $\mathrm{T}$ is widely recognized that multiple-input multiple-output 33 (MIMO) communication systems provide significant spec34 tral efficiency improvements compared with single-input35 single-output systems, owing to their higher degrees of freedom

Manuscript received September 29, 2015; revised December 25, 2015; accepted February 25, 2016. This work was supported in part by the Engineering and Physical Sciences Research Council through projects EP/Noo4558/1 and EP/L018659/1 and in part by the European Research Council's Advanced Fellow Grant through the Beam-Me-Up project and the Royal Society's Wolfson Research Merit Award. The work of N. Ishikawa was supported by the SCAT Fellowship and the MEXT/JASSO Tobitate Fellowship of Japan. The work of S. Sugiura was supported by the Japan Society for the Promotion of Science KAKENHI under Grant 26709028. The review of this paper was coordinated by Prof. H.-F. Lu.

R. Rajashekar and L. Hanzo are with the School of Electronics and Computer Science, University of Southampton, Southampton SO17 1BJ, U.K. (e-mail: rmr1u14@soton.ac.uk; 1h@ecs.soton.ac.uk).

N. Ishikawa and S. Sugiura are with the Department of Computer and Information Sciences, Tokyo University of Agriculture and Technology, Koganei 184-8588, Japan (e-mail: sugiura@ieee.org).

K. V. S. Hari is with the Department of Electrical Communication Engineering, Indian Institute of Science, Bangalore 560 012, India (e-mail: hari@ece. iisc.ernet.in).

Color versions of one or more of the figures in this paper are available online at http://ieeexplore.ieee.org.

Digital Object Identifier 10.1109/TVT.2016.2536802
[1]. However, the benefit of increased spectral efficiency comes 36 at the cost of high decoding complexity at the receiver, since 37 the transmitted symbols interfere with each other at the receiver 38 due to the simultaneous activation of multiple transmit antennas 39 (TAs). For instance, in the classic Vertical Bell Laboratories 40 Layered Space-Time architecture [2], the decoding complex- 41 ity of the maximum-likelihood (ML) receiver exponentially 42 increases with the number of TAs. An additional overhead in 43 MIMO systems is that of estimating the channel coefficients 44 between each TA and receive antenna (RA) pair and tracking 45 their changes over the entire transmission duration for coherent 46 detection [4]. Spatial modulation (SM) [5]-[8] is a beneficial 47 multiantenna scheme that overcomes some of these drawbacks. 48 Unlike the conventional MIMO system, the SM system acti- 49 vates only a single TA in each symbol duration, thereby avoid- 50 ing the interference of transmitted symbols with each other at 51 the receiver. As a further substantial benefit, it only requires a 52 single radio frequency (RF) chain, as opposed to $N_{t}$ chains, 53 albeit this potentially precludes having a transmit diversity 54 gain. More specifically, the bitstream is divided into blocks of 55 $\log _{2}\left(M N_{t}\right)$ bits, and in each block, $\log _{2}(M)$ bits are used to 56 select a symbol from an $M$-ary alphabet to be transmitted from 57 a TA chosen from $N_{t}$ TAs based on $\log _{2}\left(N_{t}\right)$ bits. 58

The SM system has been extensively studied with regard to 59 various system parameters, which include its transmit diversity 60 order [9]-[12], low-complexity near-ML detection [13]-[17], 61 TA subset selection for performance versus complexity en- 62 hancement [18]-[22], and the impact of channel estimation 63 error on the attainable performance [23]-[25]. A significant 64 research effort was spent on increasing the transmit diversity 65 order of the SM system, since achieving transmit diversity 66 gain in the SM system was not straightforward, owing to 67 the constraint of a single RF chain at the transmitter. This 68 problem was partly addressed by conceiving space-time-coded 69 SM schemes [9]-[12], which operate in an open-loop scenario, 70 and by employing TA subset selection [20], [21], which operate 71 in a closed-loop scenario. Note that both these approaches 72 require accurate channel estimation and tracking at the receiver. 73 Furthermore, the SM system has been studied in nonco- 74 herent communication scenarios [26]-[29], where the high- 75 complexity channel estimation and tracking are dispensed with 76 by employing differential encoding of the transmitted symbols. 77 Naturally, this complexity reduction is achieved at 3-dB per- 78 formance loss. This scheme is referred to as differential SM 79 (DSM) throughout this paper. More specifically, Bian et al. 80 in [26] have extended the conventional SM to a noncoherent 81 scenario by obtaining dispersion matrices (DMs) from a set of 82 
TABLE I

COMPARISON OF VARIOUS EXISTING DSM SCHEMES

\begin{tabular}{|l||c|c|c|}
\hline & P-DSM [26] & DM-DSM [27] & CS-DSM [28] \\
\hline \hline $\begin{array}{l}\text { No. of transmit } \\
\text { RF chains required }\end{array}$ & 1 & 1 & 1 \\
\hline $\begin{array}{l}\text { Throughput } \\
\text { (bpcu) }\end{array}$ & $\frac{\log _{2}\left(M^{N_{t}}\right)+\log _{2}\left\lfloor\left(N_{t} !\right)\right\rfloor_{2} p}{N_{t}}$ & $\frac{\log _{2}\left(M^{N_{t} / d}\right)+\log _{2}(Q)}{N_{t}}$ & $\frac{\log _{2}\left(Q^{\prime} Q\right)}{2}$ \\
\hline $\begin{array}{l}\text { Achievable } \\
\text { diversity } \\
\text { order }\end{array}$ & $N_{r}$ & $\begin{array}{c}d N_{r} \\
\left(1 \leq d \leq N_{t}\right)\end{array}$ & $2 N_{r}$ \\
\hline
\end{tabular}

bpcu : bits per channel use

$83\left(N_{t} \times N_{t}\right)$ permutation matrices having only a single nonzero 84 element in every row and column, where each nonzero ele85 ment is drawn from an $M$-ary phase-shift keying (PSK) signal 86 set. This scheme is referred to as permutation-based DSM 87 (P-DSM). In [27], a fixed set of sparse complex-valued DMs 88 is used in conjunction with a set of diagonal matrices, whose 89 elements are drawn from an $M$-ary PSK signal set. In this 90 scheme, a higher transmit diversity order is shown to be achiev91 able, albeit at the cost of a reduced transmission rate. We refer 92 to this scheme as DM-based DSM (DM-DSM). More recently, 93 a DM set construction was specifically proposed for two TAs 94 [28], where a transmit diversity order of 2 is guaranteed to be 95 achieved. This scheme, which employs a cyclic signal structure 96 based on diagonal matrices along with a set of fixed DMs, is 97 referred to as cyclic-signaling-based DSM (CS-DSM) in this 98 paper. Table I compares these schemes, where $Q$ denotes the 99 number of DMs, $Q^{\prime}$ represents the number of diagonal matrices 100 used for signaling [28], $d$ is the transmit diversity order, and $101\lfloor a\rfloor_{2^{p}}$ denotes the largest integer that is a power of 2 and 102 smaller than $a$, where $d$ is assumed to divide $N_{t}$ with a zero 103 remainder.

104 It is clear from Table I that the DM-DSM achieves the same 105 throughput as that of P-DSM for $d=1$ and $Q=\left\lfloor N_{t} !\right\rfloor_{2^{p}}$, 106 but this will not yield any diversity advantage. To achieve 107 the same throughput as that of P-DSM with full diversity, $108 Q$ should be equal to $M^{N_{t}-1}\left\lfloor N_{t} !\right\rfloor_{2^{p}}$. Similarly, CS-DSM is 109 capable of achieving the same throughput as that of P-DSM 110 for $Q^{\prime}=M^{N_{t}}$ and $Q=\log _{2}\left(\left\lfloor N_{t} !\right\rfloor_{2^{p}}\right)$. However, CS-DSM is 111 specifically designed for the $N_{t}=2$ case, where $Q$ has been 112 restricted to 2 [28]. Furthermore, CS-DSM is different from 113 DM-DSM in the sense that only matrices are used for encoding 114 the information bits, which is in contrast to the DM-DSM, 115 where a set of DMs and a conventional signal set are used for 116 encoding the information bits. To the best of our knowledge, 117 there is no systematic method of obtaining the number of DMs 118 required to achieve a desired throughput and transmit diversity 119 order in systems with arbitrary $N_{t}$. Hence, in this paper, we 120 focus on constructing structured DMs for DM-DSM schemes.

121 Against this background, the contributions of this paper are 122 as follows.

1) We propose a systematic method of obtaining the set of DMs for DSM systems for an arbitrary $N_{t}$ by exploiting the related results from algebraic field extensions. More specifically, we show that the companion matrix of an 127 irreducible polynomial over a certain base field will be 128 unitary, when the base field is a cyclotomic field [30], and 129 exploit these unitary companion matrices for constructing 130 DMs to be used in DSM. Additionally, we analytically 131 show that the proposed scheme is capable of achieving 132 full transmit diversity.

2) Furthermore, we generalize the proposed field-extension- 134 based DSM (FE-DSM) scheme to strike a flexible trade- 135 off between attainable diversity and multiplexing gain. 136

3) Finally, we evaluate the decoding complexity of ML 137 detection of the proposed schemes and show that they 138 offer significantly reduced complexity, owing to the DM- 139 based approach of encoding information by exploiting 140 results from [34].

The rest of this paper is organized as follows. Section II 142 provides the system model of DSM. In Section III, the proposed 143 DM set construction, as well as the diversity analysis of the pro- 144 posed scheme, are presented. Specifically, Section III-A gives 145 a brief overview of algebraic field extensions. Section III-B 146 provides the proposed DM construction and our diversity 147 analysis. In Section III-C, we conceive the low-complexity 148 decoding method for the proposed schemes. Section IV 149 provides our simulation results, and Section V concludes 150 this paper.

Notations: If $S_{1}$ and $S_{2}$ are two sets, then $S_{3}=S_{1} \times S_{2} 152$ represents the Cartesian product of sets $S_{1}$ and $S_{2}$. Lowercase 153 and uppercase boldface letters represent vectors and matrices, 154 respectively. Furthermore, $\|\cdot\|$ represents the 2-norm of a 155 vector or the Frobenius norm of a matrix. The notations of $(\cdot)^{T} 156$ and $(\cdot)^{H}$ indicate the transpose and Hermitian transpose of a 157 vector/matrix, respectively, whereas $|\cdot|$ represents the cardi- 158 nality of a given set or the magnitude of a complex quantity. 159 Furthermore, $\otimes$ defines the Kronecker product of two matrices. 160 $\mathcal{C N}\left(\mu, \sigma^{2}\right)$ denotes a complex Gaussian random variable with 161 mean $\mu$ and variance $\sigma^{2} . \mathbb{R}$ and $\mathbb{C}$ represent the field of real 162 and complex numbers, respectively. If $F$ is a field, then $F[X] 163$ represents the ring of polynomials in $X$ over $F . \mathbf{A}([a: b],:) 164$ defines a matrix with rows $a, a+1, \ldots, b-1, b$ of $\mathbf{A}$, and 165 $\mathbf{A}(:,[a: b])$ is a matrix with columns $a, a+1, \ldots, b-1, b$ of 166 A. $\mathbf{I}_{n}$ represents an $n \times n$ identity matrix. If $\mathbf{x}$ is an $n$-length 167 vector, then $\operatorname{diag}(\mathbf{x})$ represents an $n \times n$ diagonal matrix whose 168 $(i, i)$ th element is $\mathbf{x}_{i}$. 


\section{Differential Spatial Modulation System}

171 Consider a MIMO system having $N_{r}$ RAs and $N_{t}$ TAs oper172 ating in a Rayleigh flat-fading channel, which is characterized by

$$
\mathbf{Y}_{i}=\sqrt{\rho} \mathbf{H}_{i} \mathbf{X}_{i}+\mathbf{N}_{i}
$$

173 where $\mathbf{Y}_{i} \in \mathbb{C}^{N_{r} \times N_{t}}$ is the received space-time matrix (STM); $174 \mathbf{X}_{i} \in \mathbb{C}^{N_{t} \times N_{t}}$ is the transmitted STM; $\mathbf{N}_{i} \in \mathbb{C}^{N_{r} \times N_{t}}$ and $\mathbf{H}_{i} \in$ $175 \mathbb{C}^{N_{r} \times N_{t}}$ are the noise and channel matrices, respectively, whose 176 entries are from $\mathcal{C N}(0,1)$; and $\rho$ denotes the average signal-to177 noise ratio (SNR) at each RA. The subscript $i$ in all matrices 178 indicates the block index.

\section{A. DSM System}

180 Differential encoding [31], [32] of the transmitted STM is 181 given by

$$
\mathbf{X}_{i}=\mathbf{X}_{i-1} \mathbf{S}_{i}
$$

182 where $\mathbf{S}_{i} \in \mathbb{C}^{N_{t} \times N_{t}}$ is the unitary STM to be transmitted 183 during the symbol period of the $i$ th block. For the transmitted $184 \mathrm{STM} \mathrm{X}_{i}$ to become unitary, it is sufficient to ensure that $\mathbf{X}_{0}$ be 185 unitary. In this paper, we consider $\mathbf{X}_{0}$ to be $\mathbf{I}_{N_{t}}$. Furthermore, 186 each column of $\mathbf{S}_{i}$ is assumed to have only a single nonzero 187 element, since the SM system employs only a single RF chain 188 at the transmitter. Assuming that the channel remains constant 189 over a period of two successive blocks, we have

$$
\mathbf{Y}_{i-1}=\sqrt{\boldsymbol{\rho}} \mathbf{H}_{i} \mathbf{X}_{i-1}+\mathbf{N}_{i-1}
$$

190 and hence, (1) can be written as

$$
\mathbf{Y}_{i}=\mathbf{Y}_{i-1} \mathbf{S}_{i}+\mathbf{N}_{i}-\mathbf{N}_{i-1} \mathbf{S}_{i}
$$

191 Assuming that there is no channel state information at the 192 receiver, the optimal differential receiver [31] is given by

$$
\hat{\mathbf{S}}_{i}=\arg \min _{\mathbf{S} \in \mathcal{S}}\left\|\mathbf{Y}_{i}-\mathbf{Y}_{i-1} \mathbf{S}\right\|^{2}
$$

193 where $\mathcal{S}$ is the set of transmit STMs.

\section{B. DM-DSM}

195 In the case of DM-DSM, each transmitted STM is of the 196 following form:

$$
\mathbf{S}_{i}=\mathbf{D}(\mathbf{s}) \mathbf{A}_{q}
$$

197 where we have $\mathbf{s}=\left[s_{1}, s_{2}, \ldots, s_{N_{t}}\right], \mathbf{D}(\mathbf{s}) \in \mathcal{D}=\left\{\operatorname{diag}(\mathbf{s}) \mid s_{i} \in\right.$ $\left.198 \mathcal{L}_{i}-\mathrm{PSK}\right\}$, and $\mathbf{A}_{q} \in \mathcal{A}$, where $\mathcal{A}=\left[\mathbf{A}_{1}, \mathbf{A}_{2}, \ldots, \mathbf{A}_{Q}\right]$ is the 199 set of DMs. The rate achieved by DM-DSM is given by

$$
R_{\mathrm{DM}-\mathrm{DSM}}=\frac{\log _{2}\left(Q \cdot \mathcal{L}_{1} \cdots \mathcal{L}_{N_{t}}\right)}{N_{t}} \text { bpcu. }
$$

200 In the following section, we propose a method for construct201 ing the set $\mathcal{D}$ having diagonal or block-diagonal matrices as its 202 elements and the set of DMs $\mathcal{A}$, such that they enable the DSM 203 scheme to achieve full transmit diversity.
C1: We emphasize the condition that each element of $\mathcal{A} 204$ should be a unitary matrix [32] and should have only a single 205 nonzero element in each column and row. The latter condition is 206 necessary since the SM system can transmit only one symbol in 207 each channel use, owing to a single RF chain at the transmitter. 208

\section{Dispersion Matrix Set Construction}

Here, we provide a brief overview of algebraic field exten- 210 sions as required for our exposition on the proposed DM set 211 construction. For further details, see [30] and [33].

\section{A. Review of Field Extensions}

Definitions: Let $J$ be an extension of a field $L$ and $I$ be a 214 subset of $J$, i.e., $I \subset J$. Field $J$ is said to be generated by $I 215$ if $J$ is obtained by adjoining ${ }^{1}$ the elements of $I$ to $L$, and it 216 is denoted by $J=L(I)$. If set $I$ is finite, then the extension, 217 which is denoted by $J / L$, is said to be finitely generated. 218 If $\beta \in J$, then the minimal polynomial of $\beta$ is the monic 219 polynomial of least degree among the polynomials in $L[X] 220$ having $\beta$ as a root. The extended field $J$ can be viewed as 221 a vector space, where its elements are considered as vectors, 222 and the elements of $L$ are viewed as scalars. The dimension of 223 the vector space $J$ is termed as the degree of extension, and it 224 is denoted by $[J: L]$. Furthermore, the extension $J / L$ is said 225 to be an algebraic extension, if every element in $J$ is a root 226 of a nonzero polynomial with coefficients in $L$. An algebraic 227 extension $J / L$ is said to be normal if $J$ is a splitting field of 228 the family of polynomials $L[X]$, i.e., each polynomial in $L[X] 229$ splits or decomposes into linear factors over $J$. Furthermore, an 230 algebraic extension $H$ of $J$ is said to be a normal closure of 231 the algebraic extension $J / L$, if it is the only subfield of $H$ that 232 contains $J$ and if a normal extension of $L$ is $H$ itself.

233

Let $S$ be a conventional signal set, such as $M$-PSK, and $F=234$ $\mathbb{Q}(S)$ be the extended field of rationals over $S$. If $\alpha$ is a root of 235 a minimal polynomial over $F$, which is given by

$$
p(x)=x^{n}+a_{n-1} x^{n-1}+a_{n-2} x^{n-2}+\cdots+a_{0}
$$

then $F$ can be extended by adjoining $\alpha$ to obtain $K=F(\alpha) .237$ The degree of extension $[K: F]$ is equal to $n$, since $p(x)$ is 238 irreducible over $F$. Any element $k \in K$ can be expressed as 239 $\sum_{i=0}^{n-1} f_{i} \alpha^{i}$, where $f_{i} \in F \forall 0 \leq i \leq n-1$. From [30, Sec. 7.3], 240 there exists a natural mapping $k \mapsto \lambda_{k} \forall k \in K$ that embeds $K 241$ in $\mathbf{M}_{n}(F)$, where $\lambda_{k}$ is a linear transformation of $K$ into itself. 242 The regular representation of $\lambda_{k}$ maps any $v \in K$ to $k v$. The 243 linear transformation $\lambda_{\alpha}$ associated with $\alpha$ is given by

$$
\mathbf{M}=\left[\begin{array}{ccccc}
0 & 0 & \ldots & 0 & -a_{0} \\
1 & 0 & \ldots & 0 & -a_{1} \\
0 & 1 & \ldots & 0 & -a_{2} \\
\vdots & \vdots & \ddots & \vdots & \vdots \\
0 & 0 & \cdots & 1 & -a_{n-1}
\end{array}\right] \in F^{n \times n}
$$

\footnotetext{
${ }^{1}$ The adjoining operation refers to including all the elements resulting from field operations considering the elements from the extended set $I \cup L$.
} 
245 which is the companion matrix of $p(x)$. Thus, for any $k=$ $246 \sum_{i=0}^{n-1} f_{i} \alpha^{i} \in K$, the associated $\lambda_{k}$ is given by $\sum_{i=0}^{n-1} f_{i} \mathbf{M}^{i}$.

247 Lemma 1: Let $K, F$, and $S$ be defined as above. For any $248 k=k_{1}-k_{2}, k_{1} \neq k_{2} \in K, \lambda_{k} \in \mathbf{M}_{n}(F)$ is invertible.

249 Proof: The proof directly follows from $K$ being a field, 250 which guarantees the existence of the inverse for every nonzero 251 element in $K$, and the fact that the natural mapping $k \mapsto \lambda_{k}$ is 252 a one-to-one mapping.

253 Lemma 2: If $L$ is a normal closure of $K / F$ and $\sigma_{i}, i=$ $2540,1,2, \ldots, n-1$ are distinct $F$-homomorphisms from $K$ to $L$; 255 then, for any element $k \in K$, we have $\operatorname{det}\left(\lambda_{k}\right)=N_{K / F}(k)=$ $256 \prod_{i=0}^{n-1} \sigma_{i}(k)$, where $N_{K / F}(k)$ is the norm of the element $k$ from $257 K$ to $F$ [33, Th. 8].

\section{B. Proposed DM Set for DSM}

259 We propose to use the DM set given by

$$
\mathcal{A}=\left\{\mathbf{I}_{n}, \mathbf{M}, \mathbf{M}^{2}, \ldots, \mathbf{M}^{n-1}\right\}
$$

260 where $\mathbf{M}$ is as in (5), and $n$ is chosen to be equal to $N_{t}$. 261 However, to meet $C 1$, every element of $\mathcal{A}$ has to be unitary. 262 Note that it is sufficient to ensure that $\mathbf{M}$ is unitary for all 263 the elements of $\mathcal{A}$ to be unitary. Hence, we have to satisfy the 264 following equation:

$$
\mathbf{M M}^{H}=\mathbf{I}_{n} .
$$

265 Note that $\left(\mathbf{M M}^{H}\right)_{1,1}=\left|a_{0}\right|^{2}$ and $\left(\mathbf{M M}^{H}\right)_{i, i}=1+\left|a_{i-1}\right|^{2}$ for $2662 \leq i \leq n-1$. Thus, by choosing $a_{0}$ to be an element from the 267 unit circle and $a_{i}=0$ for $1 \leq i \leq n-1, C 1$ can be satisfied. 268 Thus, while constructing $\mathcal{A}$, we have to consider polynomials of 269 the form $x^{n}+a_{0}$ with $\left|a_{0}\right|=1$ values that are irreducible over $F$. 270 Since we have $|\mathcal{A}|=n=N_{t}$, our construction results in a max271 imum of $N_{t}$ DMs, i.e., $Q \leq N_{t}$. Furthermore, we assume that the 272 set $\mathcal{D}$ has scaled identity matrices of the form $s \mathbf{I}_{n}$, where $s \in S$. 273 Note that $F$ should contain the specific signal set $S$ from which $2744 s$ is chosen. Thus, the following conditions have to be met:

276 1) $S \subset F$; and

277 2) $p(x)=x^{n}+a_{0}$ with $\left|a_{0}\right|=1$ should be irreducible over $F$.

278 We satisfy the given conditions by choosing $F=\mathbb{Q}\left(S, a_{0}\right)$, 279 where $a_{0}$ is any transcendental element over $\mathbb{Q}(S)$ lying on the 280 unit circle. In the following, we shall explain the method of 281 constructing set $\mathcal{A}$ in detail.

282 Let $S$ be a conventional $M$-PSK signal set denoted by $283\left\{\omega_{M}^{i}\right\}_{i=0}^{M-1}$, where we have $\omega_{M}=e^{j 2 \pi / M}$ and $a_{0}=-e^{j u_{1}}$, 284 with $u_{1}$ being algebraic over $\mathbb{Q}$. For instance, $u_{1}$ can be $\sqrt{3}$, 285 which is a root of the polynomial $x^{2}-3$. Note that $a_{0}$ is 286 transcendental over $\mathbb{Q}(S)$, and we can choose $F=\mathbb{Q}\left(S, e^{j u_{1}}\right)$. 287 Thus, the polynomial $x^{n}+a_{0}=x^{n}-e^{j u_{1}}$ (for any $n$ ) is ir288 reducible over $F$. Therefore, we can have the extension $K=$ $289 F(\alpha)$, where $\alpha$ is the primitive $n$th root of $e^{j u_{1}}$. Thus, the 290 associated companion matrix is given by

$$
\mathbf{M}=\left[\begin{array}{ccccc}
0 & 0 & \cdots & 0 & e^{j u_{1}} \\
1 & 0 & \cdots & 0 & 0 \\
0 & 1 & \cdots & 0 & 0 \\
\vdots & \vdots & \ddots & \vdots & \vdots \\
0 & 0 & \cdots & 1 & 0
\end{array}\right] \in F^{n \times n} .
$$

Example 1: Consider $n=N_{t}=4$ and $a_{0}=-e^{j \sqrt{3}}$. Then, 291 the elements of set $\mathcal{A}$ are given by $\mathbf{I}_{4}$

$$
\begin{aligned}
\mathbf{M} & =\left[\begin{array}{cccc}
0 & 0 & 0 & e^{j \sqrt{3}} \\
1 & 0 & 0 & 0 \\
0 & 1 & 0 & 0 \\
0 & 0 & 1 & 0
\end{array}\right], \mathbf{M}^{2}=\left[\begin{array}{cccc}
0 & 0 & e^{j \sqrt{3}} & 0 \\
0 & 0 & 0 & e^{j \sqrt{3}} \\
1 & 0 & 0 & 0 \\
0 & 1 & 0 & 0
\end{array}\right] \\
\mathbf{M}^{3} & =\left[\begin{array}{cccc}
0 & e^{j \sqrt{3}} & 0 & 0 \\
0 & 0 & e^{j \sqrt{3}} & 0 \\
0 & 0 & 0 & e^{j \sqrt{3}} \\
1 & 0 & 0 & 0
\end{array}\right] .
\end{aligned}
$$

Remark 1: Note that $a_{0}$ has to be chosen in conjunction with 293 the specific signal set $S$ that maximizes a certain performance 294 metric, such as the coding gain. This can be achieved by 295 searching for an optimal $a_{0}$ over a large set of closely spaced 296 transcendental elements on the unit circle.

297

We term the DSM scheme employing the proposed FE-DMs 298 as an FE-DSM scheme. Since the set of transmit STMs is given 299 by $\mathcal{S}=\mathcal{D} \times \mathcal{A}$, the rate achieved by the proposed scheme is $\quad 300$

$$
\begin{aligned}
R_{\mathrm{FE}-\mathrm{DSM}} & =\frac{\log _{2}(|\mathcal{D} \| \mathcal{A}|)}{N_{t}} \\
& =\frac{\log _{2}\left(M N_{t}\right)}{N_{t}} \mathrm{bpcu} .
\end{aligned}
$$

1) Diversity Gain: The achievable transmit diversity order 301 under differential detection [31, Sec. III-C] of (2) is given by 302

$$
d=\min _{\mathbf{S}_{1} \neq \mathbf{S}_{2} \in \mathcal{S}} \operatorname{rank}\left(\mathbf{S}_{1}-\mathbf{S}_{2}\right) .
$$

Proposition 1: The proposed FE-DSM scheme achieves a 303 transmit diversity order of $N_{t}$, i.e., $d=N_{t}$.

Proof: The proof is given in Appendix A.

2) Coding Gain: The coding gain of the proposed scheme is 306 given by

$$
G=\min _{\mathbf{S}_{1} \neq \mathbf{S}_{2} \in \mathcal{S}}\left|\operatorname{det}\left[\left(\mathbf{S}_{1}-\mathbf{S}_{2}\right)\left(\mathbf{S}_{1}-\mathbf{S}_{2}\right)^{H}\right]\right|^{\frac{1}{n}} .
$$

In the following, we shall provide a simple expression for 308 the determinant term in (10) that allows us to optimize the 309 exponential $a_{0}$ in conjunction with an arbitrary $M$-PSK signal 310 set to achieve a high coding gain.

311

Proposition 2: Consider an FE-DSM system using an $M$-PSK 312 signal set and $N_{t}=n$ TAs. If $\mathbf{S}=e^{j(2 \pi p / M)} \mathbf{M}^{l}$ and $\mathbf{S}_{2}=313$ $e^{j(2 \pi q / M)} \mathbf{M}^{m}$, where $0 \leq p, q \leq M-1$ and $0 \leq l, m \leq n-1314$ such that $\mathbf{S}_{1} \neq \mathbf{S}_{2}$, then $\left|\operatorname{det}\left[\left(\mathbf{S}_{1}-\mathbf{S}_{2}\right)\left(\mathbf{S}_{1}-\mathbf{S}_{2}\right)^{H}\right]\right|$ is given by 315

$$
4^{n} \prod_{r=0}^{n-1} \sin ^{2}\left(\frac{\pi(p-q)}{M}+\frac{\left(2 \pi r+u_{1}\right)(m-l)}{2 n}\right) .
$$

Proof: The proof is provided in Appendix B.

In the following section, we provide a DM set construction 317 based on two levels of field extensions, which facilitate a 318 flexible tradeoff between the attainable transmit diversity and 319 multiplexing gain. 


\section{C. FE-DSM With Diversity-Rate Tradeoff}

322 The DM set construction presented in the previous section 323 achieves a transmit diversity order of $N_{t}$, while transmitting 324 only a single symbol from an $M$-PSK signal set. Note that when 325 the channel conditions are good, it may not be necessary to 326 exploit the full transmit diversity order. Under these conditions, 327 we may aim at trading off the diversity gain for increasing 328 the transmission rate. In the following, we shall provide a 329 systematic method of constructing a DM set that achieves 330 the desired diversity order and transmission rate. The DM set 331 construction presented in the previous section may be viewed 332 as a special case.

333 Let $N_{t}$ be factored as $g \cdot h$. We construct a DM set that allows 334 us to transmit $h$ independent $M$-PSK symbols in each transmit 335 STM and achieve transmit diversity order $g$. Considering $F=$ $336 \mathbb{Q}\left(S,-e^{j u_{1}}\right)$ as before and the extension $K=F(\alpha)$, where $\alpha$ 337 is a primitive $g$ th root of the polynomial $p_{1}(x)=x^{g}-e^{j u_{1}}$, we 338 obtain the DM set given by

$$
\mathcal{A}^{\prime}=\left\{\mathbf{I}_{g}, \mathbf{M}, \mathbf{M}^{2}, \ldots, \mathbf{M}^{g-1}\right\}
$$

339 where $\mathbf{M} \in F^{g \times g}$ is the companion matrix of $p_{1}(x)$. We define $340 \mathcal{D}$ to be a set of block-diagonal matrices given by

$$
\mathcal{D}=\left\{\operatorname{diag}\left(s_{1} \mathbf{A}_{1}, s_{2} \mathbf{A}_{2}, \ldots, s_{h} \mathbf{A}_{h}\right) \mid s_{i} \in M-\mathrm{PSK}, \mathbf{A}_{i} \in \mathcal{A}^{\prime}, \forall i\right\} .
$$

341 Let us now consider the field extension $L=K(\beta)$ associated 342 with the polynomial $p_{2}(x)=x^{h}-e^{j u_{2}}$, where $e^{j u_{2}}$ is tran343 scendental over $K$, and $\beta$ is the primitive $h$ th root of $e^{j u_{2}}$. Then, 344 the regular representation of an element $l=\sum_{i=0}^{h-1} k_{i} \beta^{i} \in L$ 345 is given by $\sum_{i=0}^{h-1} k_{i} \mathbf{N}^{i}$, where $k_{i} \in K, 0 \leq i \leq h-1$, and $346 \mathbf{N} \in K^{h \times h}$ is the companion matrix of $p_{2}(x)$. We define the 347 DM set as

$$
\mathcal{A}=\left\{\mathbf{I}_{n}, \mathbf{N}^{\prime}, \mathbf{N}^{\prime 2}, \ldots, \mathbf{N}^{\prime h-1}\right\}
$$

348 where $\mathbf{N}^{\prime}=\mathbf{N} \otimes \mathbf{I}_{g}$. The transmit $\mathrm{STM}$ set is given by $349 \mathcal{S}=\mathcal{D} \times \mathcal{A}$ as before. We refer to this scheme as the FE-DSM 350 arrangement exhibiting a flexible diversity-rate tradeoff (FE351 DSM-DR). Note that the DSM scheme requires each transmit 352 STM to be unitary. The following proposition shows that this 353 condition is satisfied.

354 Proposition 3: If $\mathcal{S}$ is the set of transmit STMs of FE-DSM$355 \mathrm{DR}$, then each element in $\mathcal{S}$ is unitary.

356 Proof: The proof is provided in Appendix C.

357 In the following, we shall provide an example construction 358 to further illustrate the given set of points.

359 Since we have $|\mathcal{D}|=(M g)^{h}$ and $|\mathcal{A}|=h$, the rate achieved 360 by the FE-DSM-DR is given by

$$
R_{\mathrm{FE}-\mathrm{DSM}-\mathrm{DR}}=\frac{h \log _{2}(M g)+\log _{2}(h)}{N_{t}} \text { bpcu. }
$$

361 Note that when we have $g=N_{t}$, FE-DSM-DR reduces to the 362 FE-DSM scheme.
Example 2: Let $n=N_{t}=4, g=h=2, u_{1}=\sqrt{2}$, and 363 $u_{2}=\sqrt{3}$. The elements of set $\mathcal{D}$ are

364

$$
\begin{aligned}
& {\left[\begin{array}{cccc}
s_{1} & 0 & 0 & 0 \\
0 & s_{1} & 0 & 0 \\
0 & 0 & s_{2} & 0 \\
0 & 0 & 0 & s_{2}
\end{array}\right],\left[\begin{array}{cccc}
0 & s_{1} e^{j \sqrt{2}} & 0 & 0 \\
s_{1} & 0 & 0 & 0 \\
0 & 0 & s_{2} & 0 \\
0 & 0 & 0 & s_{2}
\end{array}\right]} \\
& {\left[\begin{array}{cccc}
0 & s_{1} e^{j \sqrt{2}} & 0 & 0 \\
s_{1} & 0 & 0 & 0 \\
0 & 0 & 0 & s_{2} e^{j \sqrt{2}} \\
0 & 0 & s_{2} & 0
\end{array}\right],\left[\begin{array}{cccc}
s_{1} & 0 & 0 & 0 \\
0 & s_{1} & 0 & 0 \\
0 & 0 & 0 & s_{2} e^{j \sqrt{2}} \\
0 & 0 & s_{2} & 0
\end{array}\right]}
\end{aligned}
$$

where $s_{1}$ and $s_{2}$ are from the classic $M$-PSK signal set. The 365 elements of the DM set $\mathcal{A}$ are

366

$$
\left[\begin{array}{cccc}
1 & 0 & 0 & 0 \\
0 & 1 & 0 & 0 \\
0 & 0 & 1 & 0 \\
0 & 0 & 0 & 1
\end{array}\right],\left[\begin{array}{cccc}
0 & 0 & e^{j \sqrt{3}} & 0 \\
0 & 0 & 0 & e^{j \sqrt{3}} \\
1 & 0 & 0 & 0 \\
0 & 1 & 0 & 0
\end{array}\right]
$$

Remark 2: Note that $e^{j u_{1}}$ and $e^{j u_{2}}$ have to be optimized 367 in conjunction with the signal set $S$ to maximize the coding 368 gain. Unlike FE-DSM, the STM matrices of the FE-DSM-DR 369 scheme are not representations of field elements, and hence, no 370 closed-form expression is derived for the determinant of the 371 codeword difference matrix. We resort to numerical search to 372 arrive at the optimal values of $u_{1}$ and $u_{2}$.

373

\section{ML Decoding Complexity}

Here, we evaluate the complexity order of ML decoding 375 for the proposed schemes. We show that the ML decoding 376 complexity of both proposed schemes is independent of the size 377 of the signal set $S$.

1) FE-DSM: Let $\chi=\left\{s \mathbf{e}_{i} \mid 1 \leq i \leq Q, s \in S\right\}$, where $\mathbf{e}_{i}$ is 379 the $i$ th column of $\mathbf{I}_{Q}$. Furthermore, let $\mathbf{G}=\left[\operatorname{vec}\left(\mathbf{A}_{1}\right), \operatorname{vec}\left(\mathbf{A}_{2}\right), 380\right.$ $\left.\ldots, \operatorname{vec}\left(\mathbf{A}_{Q}\right)\right] \in \mathbb{C}^{N_{t}^{2} \times Q}$, where $\mathbf{A}_{i}$ values are the elements of $\mathcal{A}$. 381 Considering the optimal detection rule of (2), we have

$$
\begin{aligned}
\hat{\mathbf{S}}_{i} & =\arg \min _{\mathbf{S} \in \mathcal{S}}\left\|\mathbf{Y}_{i}-\mathbf{Y}_{i-1} \mathbf{S}\right\|^{2} \\
& \equiv \arg \min _{s \in S, \mathbf{A}_{q} \in \mathcal{A}}\left\|\mathbf{Y}_{i}-\mathbf{Y}_{i-1}\left(s \mathbf{A}_{q}\right)\right\|^{2} \\
& \equiv \arg \min _{\mathbf{s} \in \chi}\left\|\overline{\mathbf{Y}}_{i}-\left(\mathbf{I}_{N_{t}} \otimes \mathbf{Y}_{i-1}\right) \mathbf{G} \mathbf{s}\right\|^{2}
\end{aligned}
$$

where $\overline{\mathbf{Y}}_{i}=\operatorname{vec}\left(\mathbf{Y}_{i}\right) \in \mathbb{C}^{N_{r} N_{t} \times 1}$. Since we have $|\chi|=Q|S|, 383$ the decoding complexity order is $\mathcal{O}(Q M)$, when $S$ is an 384 $M$-PSK signal set. However, owing to the interference-free 385 nature of transmit vectors, the decoding complexity can be 386 reduced from $\mathcal{O}(Q M)$ to $\mathcal{O}(Q)$ with the aid of hard-limiting 387 (HL)-based detection [34]. In other words, the ML decoding 388 complexity of the FE-DSM scheme does not scale with the size 389 of the signal set. By contrast, the existing full-diversity DSM 390 scheme in [28] does not allow such low decoding complexity. 391 
2) FE-DSM-DR: The optimal detection rule of (2) yields

$$
\begin{aligned}
\hat{\mathbf{S}}_{i} & =\arg \min _{\mathbf{S} \in \mathcal{S}}\left\|\mathbf{Y}_{i}-\mathbf{Y}_{i-1} \mathbf{S}\right\|^{2} \\
& \equiv \arg \min _{\mathbf{D} \in \mathcal{D}, \mathbf{A}_{q} \in \mathcal{A}}\left\|\mathbf{Y}_{i}-\mathbf{Y}_{i-1} \mathbf{D} \mathbf{A}_{q}\right\|^{2} \\
& \equiv \arg \min _{0 \leq k \leq h-1}\left\{\min _{\mathbf{D} \in \mathcal{D}}\left\|\mathbf{Y}_{i}-\mathbf{Y}_{i-1} \mathbf{D} \mathbf{N}^{\prime k}\right\|^{2}\right\} \\
\left(\hat{k}, \hat{\mathbf{D}}^{(\hat{k})}\right) & \equiv \arg \min _{0 \leq k \leq h-1}\left\|\mathbf{Z}_{i}^{(k)}-\mathbf{Y}_{i-1} \hat{\mathbf{D}}^{(k)}\right\|^{2}
\end{aligned}
$$

393 where $\hat{\mathbf{D}}^{(k)}=\min _{\mathbf{D} \in \mathcal{D}}\left\|\mathbf{Z}_{i}^{(k)}-\mathbf{Y}_{i-1} \mathbf{D}\right\|^{2}$, and $\mathbf{Z}_{i}^{(k)}=\mathbf{Y}_{i}\left(\mathbf{N}^{\prime k}\right)^{H}$ 394 for $0 \leq k \leq h-1$. Since $\mathbf{D}$ is block diagonal, we have

$$
\begin{aligned}
\hat{\mathbf{D}}^{(k)} & =\min _{\mathbf{D} \in \mathcal{D}}\left\|\mathbf{Z}_{i}^{(k)}-\mathbf{Y}_{i-1} \mathbf{D}\right\|^{2} \\
& \equiv \sum_{l=1}^{h} \min _{s_{l} \in S, \mathbf{A}_{i_{l}} \in \mathcal{A}^{\prime}}\left\|\mathbf{Z}_{i}^{(k)}\left(:, \mathcal{I}_{l}\right)-\mathbf{Y}_{i-1}\left(:, \mathcal{I}_{l}\right)\left(s_{l} \mathbf{A}_{i_{l}}\right)\right\|^{2}
\end{aligned}
$$

395 where $\mathcal{I}_{l}=[g(l-1)+1: g l]$. By invoking the HL-based 396 detector in [34], the search complexity of the minimization 397 problem, i.e.,

$$
\min _{s_{l} \in S, \mathbf{A}_{i_{l}} \in \mathcal{A}^{\prime}}\left\|\mathbf{Z}_{i}^{(k)}\left(:, \mathcal{I}_{l}\right)-\mathbf{Y}_{i-1}\left(:, \mathcal{I}_{l}\right)\left(s_{l} \mathbf{A}_{i_{l}}\right)\right\|^{2}
$$

398 can be reduced from $\mathcal{O}\left(|S|\left|\mathcal{A}^{\prime}\right|\right)$ to $\mathcal{O}\left(\left|\mathcal{A}^{\prime}\right|\right)=\mathcal{O}(g)$. Specifi399 cally, this is achieved by converting (25) into an interference400 free system analogous to (18) and then employing the detector 401 in [34]. Thus, the ML decoding complexity order of FE-DSM$402 \mathrm{DR}$ is independent of the size of the signal set, and it is given 403 by $\mathcal{O}\left(\left|\mathcal{A}^{\prime}\right||\mathcal{A}|\right)=\mathcal{O}(g h)=\mathcal{O}\left(N_{t}\right)$.

\section{E. Computational Complexity}

405 Here, we compare the computational complexity of the ML 406 detector of various existing schemes with that of the proposed 407 scheme. Specifically, we show that all the existing schemes 408 essentially impose the same computational complexity when 409 operating at a given rate. However, since the ML decoding 410 complexity order of the proposed schemes does not scale with 411 the signal set, the computational complexity involved in ML 412 decoding remains constant, when the size of the signal set is 413 increased to increase the transmission rate.

414 Considering the ML detection rule of (2), we have

$$
\hat{\mathbf{S}}_{i}=\arg \min _{\mathbf{S} \in \mathcal{S}}\left\|\mathbf{Y}_{i}-\mathbf{Y}_{i-1} \mathbf{S}\right\|^{2}
$$

415 where $\mathcal{S}$ is the set of transmit STMs. The number of real-valued 416 multiplications in evaluating (26) is $6 N_{r} N_{t}|\mathcal{S}|$, where $|\mathcal{S}|$ is the 417 cardinality of the set of transmit STMs. When the transmission 418 rate is fixed, $|\mathcal{S}|$ is essentially the same across all the existing 419 schemes [26]-[28]. The direct evaluation of (26) results in the 420 same computational complexity across all the schemes, since 421 the number of nonzero elements in each $\mathbf{S} \in \mathcal{S}$ is the same 422 in all of them. However, the proposed FE-DSM (DM-DSM 423 [27]) scheme has the property that $\mathcal{S}=S \times \mathcal{A}$, which makes

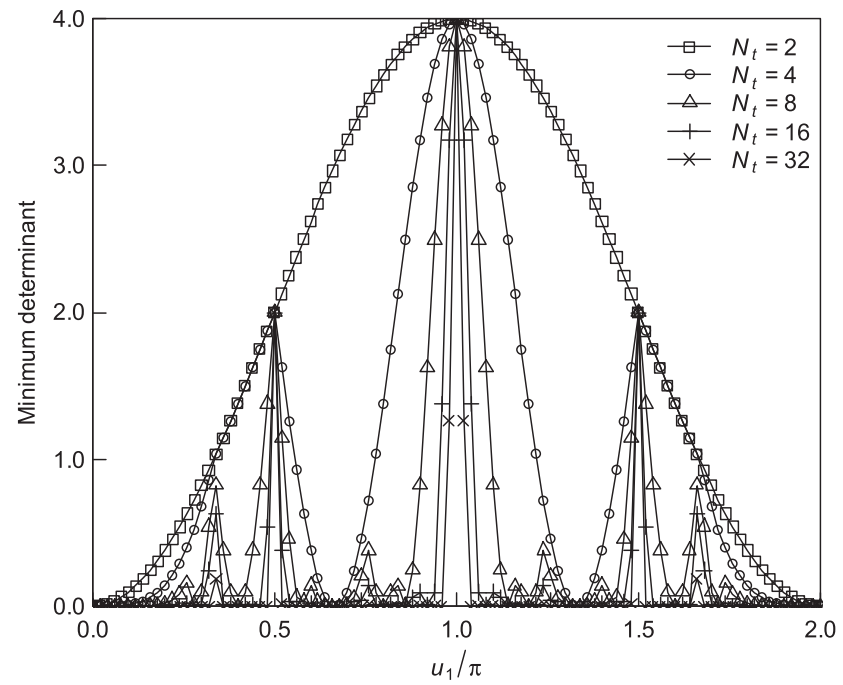

Fig. 1. Variation of coding gain as a function of $u_{1}$ in FE-DSM employing a BPSK signal set for various $N_{t}$ values.

it amenable to HL-based ML detection (HL-ML) [34]. The 424 computational complexity imposed by the HL-ML detector can 425 be shown to be $\left(10 N_{t} N_{r}+9\right)|\mathcal{A}| .^{2}$ In the following section, we 426 compare the computational complexity imposed by the direct 427 ML solution in (26) to that of the HL-ML solution [34] by 428 considering various system parameters and transmission rates. 429

\section{Simulation Results and Discussions}

430

Simulation Parameters: In all our simulations, we have used 431 block Rayleigh fading channels. In evaluating the bit error ratio 432 (BER) of $10^{-t}$, we have used at least $10^{t+2}$ bits. For DM-DSM 433 schemes operating at different rates, the optimal DM sets are 434 obtained by optimizing the coding gain over a large set of 435 feasible matrices in conjunction with the associated $M$-PSK 436 signal set. The parameter $e^{j u_{1}}$ of FE-DSM and the parameters 437 $\left(e^{j u_{1}}, e^{j u_{2}}\right)$ of FE-DSM-DR are optimized in conjunction with 438 the associated signal sets to obtain the optimal set of DMs. For 439 the FE-DSM scheme using an $M$-PSK signal set, it is observed 440 that $u_{1}=2 \pi / M$ is optimal for any value of $N_{t}$. Fig. 1 shows 441 the achievable coding gain of FE-DSM employing a binary 442 phase-shift keying (BPSK) signal set. It is clear in Fig. 1 that 443 the value of $u_{1}=\pi$ remains optimal even when $N_{t}$ is varied. 444

Fig. 2 compares the BER performance of the FE-DSM and 445 DM-DSM schemes, both having $N_{t}=2$ and employing 4-PSK 446 as well as 16-PSK signal sets that achieve a throughput of 1.5447 and $2.5 \mathrm{bpcu}$, respectively. The BER performance of P-DSM is 448 also provided to highlight the transmit diversity gain achieved 449 by the DM-DSM scheme. Furthermore, the BER performance 450 of the proposed codebooks in the coherent scenario is also pro- 451 vided. Fig. 3 compares the BER performance of the FE-DSM 452 and DM-DSM schemes, both having $N_{t}=4$ and employing 453 4-PSK, as well as 16-PSK signal sets that achieve a throughput 454 of 1 and $1.5 \mathrm{bpcu}$, respectively. It is clear in Figs. 2 and 3 that 455

\footnotetext{
${ }^{2}$ It takes $4 N_{t} N_{r}|\mathcal{A}|$ multiplications to compute $\left(\mathbf{I}_{N_{t}} \otimes \mathbf{Y}_{i-1}\right) \mathbf{G}$ and $\left(6 N_{t} N_{r}+9\right)|\mathcal{A}|$ multiplications to compute various decision metrics of the HL-ML detector [34]. For further details, see [34, Sec. IV-B]
} 


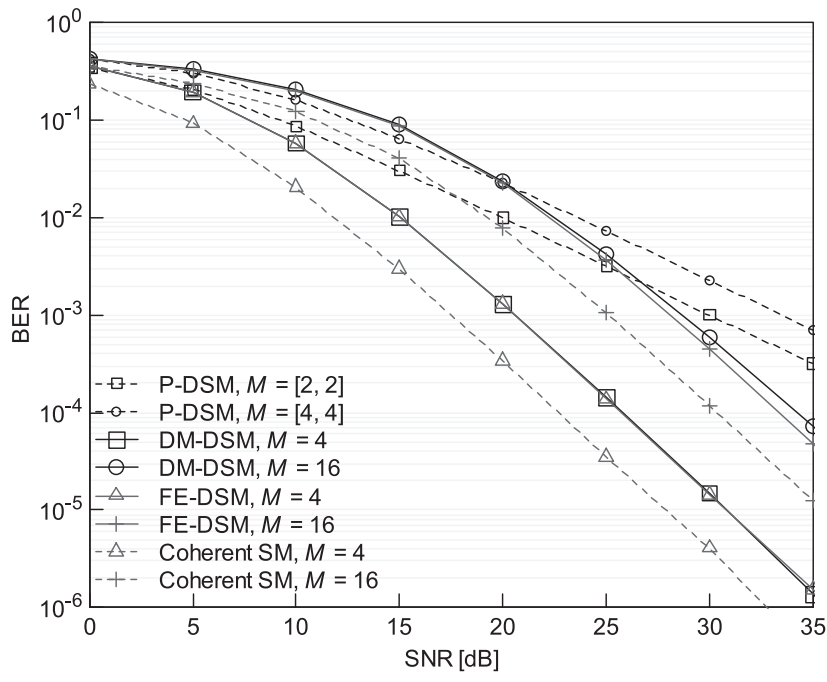

Fig. 2. BER performance of the DM-DSM and FE-DSM schemes, having $N_{t}=2$ and employing 4-PSK and 16-PSK signal sets. The BER performance of the P-DSM scheme is provided to highlight the transmit diversity gain achieved in DM-DSM and FE-DSM.

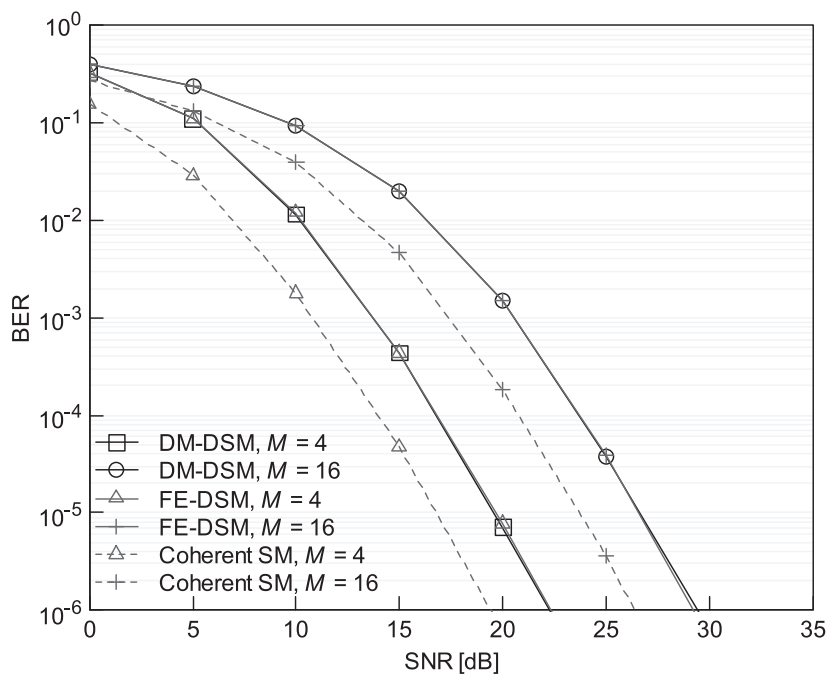

Fig. 3. BER performance of the DM-DSM and FE-DSM schemes, having $N_{t}=4$ and employing $M$-PSK signal sets.

456 the proposed FE-DSM scheme suffers from no performance 457 loss compared with the DM-DSM scheme, and there is a 3$458 \mathrm{~dB}$ performance loss with respect to the coherent counterparts, 459 which is as expected.

460 Fig. 4 compares the BER performance of FE-DSM-DR and 461 DM-DSM that trades off diversity gain against throughput. 462 Both the systems are assumed to have $N_{t}=4$. Specifically, four 463 data rates are considered for comparison. For $h=g=2$, FE464 DSM-DR achieves throughput values of $1.25,1.75,2.25$, and 4652.75 bpcu when employing BPSK, quaternary phase-shift key466 ing, 8-PSK, and 16-PSK signal sets, respectively. The DM467 DSM scheme is assumed to have a set of four DMs as proposed 468 in [27] and employs $M=\left[\left(L_{1}, L_{1}\right),\left(L_{2}, L_{2}\right)\right]$, where $L_{1}$ and $469 L_{2}$ correspond to the sizes of the PSK signal sets encoding sym470 bols $s_{1}$ and $s_{2}$, respectively. To elaborate, $L_{1}$ and $L_{2}$ are chosen 471 so that the rates achieved by the proposed scheme and the 472 FE-DSM scheme are the same. When operating at $1.25 \mathrm{bpcu}$,

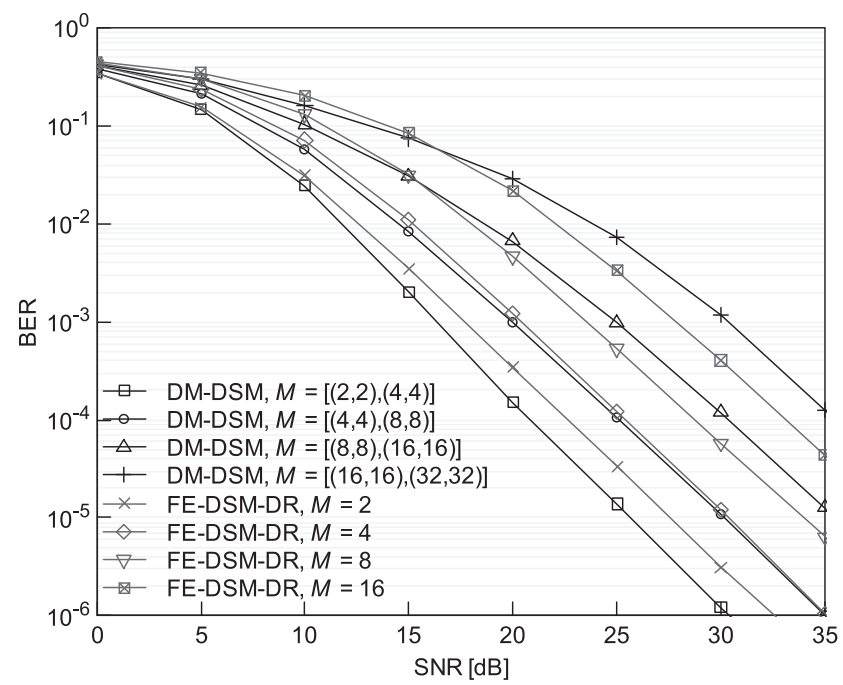

Fig. 4. BER performance of the DM-DSM and FE-DSM-DR schemes, having $N_{t}=4$ and employing $M$-PSK signal sets.
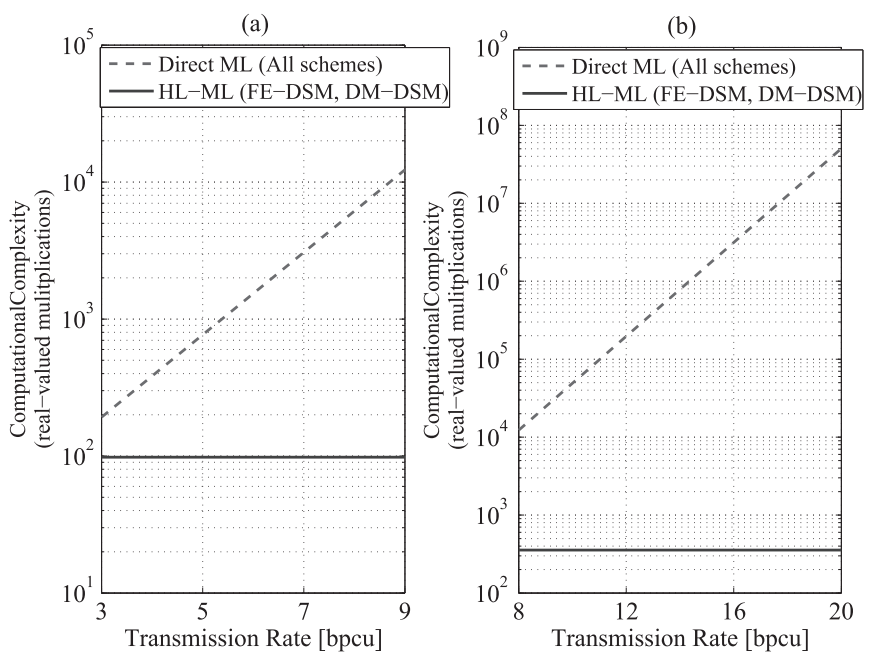

Fig. 5. Comparison of the computational complexity imposed by the ML detector in the existing schemes with that of the proposed scheme in systems having $N_{t}=2,4$ and $N_{r}=2$ and employing various transmission rates.

it can be observed in Fig. 4 that FE-DSM-DR suffers from a 473 1.5-dB SNR loss at a BER of $10^{-4}$ compared with DM-DSM. 474 However, as the rate is increased from 1.25 to $2.75 \mathrm{bpcu}$, the 475 performance of FE-DSM-DR improves, which is evident in 476 Fig. 4. Specifically, when operating at 2.25 and $2.75 \mathrm{bpcu}$, it is 477 observed that the FE-DSM-DR scheme achieves an SNR gain 478 of about $1 \mathrm{~dB}$ and about $2 \mathrm{~dB}$ at a BER of $10^{-4}$, respectively. 479

Fig. 5 gives the computational complexity imposed by the 480 ML detector in various existing schemes along with that of the 481 proposed FE-DSM scheme in systems having $N_{t}=2,4$ and 482 $N_{r}=2$ and employing various transmission rates. In the case 483 of $N_{t}=2$, the P-DSM scheme is assumed to employ BPSK, 484 4-PSK, 8-PSK, and 16-PSK to achieve a transmission rate of 485 $3,5,7$, and $9 \mathrm{bpcu}$, respectively, and in the case of $N_{t}=4,486$ the same signal sets achieve a transmission rate of $8,12,16,487$ and $20 \mathrm{bpcu}$, respectively. In the case of FE-DSM, the size 488 of the DM set is fixed to 2 and 4 in the case of $N_{t}=2$ and 489 $N_{t}=4$, respectively, and the size of the signal set (or the 490 
491 number of cyclic matrices in the case of CS-DSM [26] when $492 N_{t}=2$ ) is assumed to vary to increase the transmission rate. It 493 is clear in Fig. 5 that the HL-ML detector results in significant 494 complexity reductions over the direct ML solution. Specifically, 495 at a transmission rate of 5 and $8 \mathrm{bpcu}$, a reduction of about 496670 multiplications in the case of $N_{t}=2$ and about 11934 497 multiplications in the case of $N_{t}=4$ is observed, respectively. 498 Future Work: While the proposed DM set constructions cater 499 to the requirements of the SM scheme relying on differential 500 encoding, it would be interesting to study the feasibility of ex501 tending the proposed schemes to generalized SM (GSM) [35], 502 where more than one TAs are activated during each channel use. 503 Note, however, that this is not straightforward, since

504

505 1) the transmitted STMs in GSM may not be unitary in 506 general; and

507 2) the product of any two distinct transmit STMs does not 508 satisfy the sparsity constraint analogous to condition C1 509 given in Section II-B.

510 As inferred from Fig. 4, the FE-DSM-DR-based DMs are not 511 optimal at low rates (unlike the FE-DSM scheme). Furthermore, 512 considering algebraic structures for designing DM sets would 513 enable us to quantify the achievable diversity order and the cod514 ing gain in addition to attaining the benefits of a simple and sys515 tematic encoding at the transmitter. Thus, worth investigating 516 are other representations of algebraic structures such as division 517 algebras for their suitability in obtaining sparse, full-diversity, 518 and optimal DM sets for differential SM/GSM schemes.

\section{CONCLUSION}

520 We have proposed a systematic method for obtaining a DM 521 set for DSM with the aid of algebraic field extensions. It was 522 analytically shown that the proposed FE-DSM achieves full 523 transmit diversity. Furthermore, a closed-form expression was 524 derived for the determinant of the codeword difference matrix. 525 The proposed FE-DSM scheme was then further extended to 526 FE-DSM-DR, which stroke a flexible tradeoff between diver527 sity gain and throughput. Both the proposed schemes were 528 shown to offer ML decoding complexity, which is independent 529 of the size of the signal set. Our simulation results have shown 530 that the FE-DSM scheme achieves the same BER performance 531 as the DM-DSM scheme, whereas FE-DSM-DR is observed to 532 give a better BER performance at higher rates compared with 533 its DM-DSM counterpart.

\section{4 \\ 535 \\ PROOF OF PROPOSITION 1}

536 Let $\mathbf{S}_{1}=s \mathbf{M}^{i}$ and $\mathbf{S}_{2}=s^{\prime} \mathbf{M}^{j}$, where $s, s^{\prime} \in S \subset F=$ $537 \mathbb{Q}\left(S, a_{0}\right)$. Recall that $\mathbf{S}_{1}$ and $\mathbf{S}_{2}$ are regular representations 538 of $k_{1}=s \alpha^{i}$ and $k_{2}=s^{\prime} \alpha^{j}$, respectively. Then, we have $\lambda_{k}=$ $539 \mathbf{S}_{1}-\mathbf{S}_{2}$, where $k=k_{1}-k_{2}$. From Lemma 1 , we see that $\lambda_{k}$ is 540 invertible for all $k_{1}, k_{2} \in K$ and $k_{1} \neq k_{2}$. Thus, we have $\mathbf{S}_{1}-$ $541 \mathbf{S}_{2}$ as invertible. In other words, we have $\operatorname{rank}\left(\mathbf{S}_{1}-\mathbf{S}_{2}\right)=n=$ $542 N_{t}, \forall \mathbf{S}_{1} \neq \mathbf{S}_{2} \in \mathcal{S}$. Thus, we have $\min _{\mathbf{S}_{1} \neq \mathbf{S}_{2} \in \mathcal{S}} \operatorname{rank}\left(\mathbf{S}_{1}-\right.$ $\left.543 \mathbf{S}_{2}\right)=N_{t}$. This concludes the proof.
APPENDIX B

PROOF OF PROPOSITION 2

If $\lambda_{k}=\mathbf{S}_{1}-\mathbf{S}_{2}$, then the element $k$ associated with $\lambda_{k}$ is given 546 by $s \alpha^{m}-s^{\prime} \alpha^{l}$, where $s=e^{j(2 \pi p / M)}, s^{\prime}=e^{j(2 \pi q / M)}$, and $\alpha$ is the 547 primitive $n$th root of $-a_{0}$. From Lemma 2, we have $\operatorname{det}\left(\lambda_{k}\right)=548$ $\operatorname{det}\left(\mathbf{S}_{1}-\mathbf{S}_{2}\right)=\prod_{r=0}^{n-1} \sigma_{r}(k)$, where we have $\sigma_{r}: \alpha \mapsto \alpha_{r}, 549$ such that $\alpha_{r}, 0 \leq r \leq n-1$ are the $n$th roots of $-a_{0}=e^{j u_{1}} .550$ Therefore, we have

$$
\begin{aligned}
\operatorname{det}\left(\mathbf{S}_{1}-\mathbf{S}_{2}\right) & =\prod_{r=0}^{n-1} \sigma_{r}(k)=\prod_{r=0}^{n-1} \sigma_{r}\left(s \alpha^{m}-s^{\prime} \alpha^{l}\right) \\
& =\prod_{r=0}^{n-1}\left(s\left(\sigma_{r}(\alpha)\right)^{m}-s^{\prime}\left(\sigma_{r}(\alpha)\right)^{l}\right) \\
& =\prod_{r=0}^{n-1}\left(e^{j \frac{2 \pi p}{M}+\frac{\left(2 \pi r+u_{1}\right) m}{n}}-e^{j \frac{2 \pi q}{M}+\frac{\left(2 \pi r+u_{1}\right) l}{n}}\right) .
\end{aligned}
$$

Thus, we have

$$
\begin{aligned}
\operatorname{det} & {\left[\left(\mathbf{S}_{1}-\mathbf{S}_{2}\right)\left(\mathbf{S}_{1}-\mathbf{S}_{2}\right)^{H}\right] } \\
& =\mid \prod_{r=0}^{n-1}\left(e^{j \frac{2 \pi p}{M}+\frac{\left(2 \pi r+u_{1}\right) m}{n}}-\left.e^{\left.j \frac{2 \pi q}{M}+\frac{\left(2 \pi r+u_{1}\right) l}{n}\right)}\right|^{2}\right. \\
& =4^{n} \prod_{r=0}^{n-1} \sin ^{2}\left(\frac{\pi(p-q)}{M}+\frac{\left(2 \pi r+u_{1}\right)(m-l)}{2 n}\right) .
\end{aligned}
$$

This concludes the proof.

\section{APPENDIX C}

Let $\mathbf{S} \in \mathcal{S}$ such that $\mathbf{S}=\operatorname{diag}\left(s_{1} \mathbf{A}_{1}, s_{2} \mathbf{A}_{2}, \ldots, s_{h} \mathbf{A}_{h}\right) \mathbf{N}^{\prime k} 556$ for some $0 \leq k \leq h-1, s_{i} \in M$-PSK signal set, $\mathbf{A}_{i} \in \mathcal{A}^{\prime}$ for 557 $1 \leq i \leq h$. Consider $\mathbf{S S}^{H}=\mathbf{D} \mathbf{N}^{\prime k} \mathbf{N}^{\prime k} \mathbf{D}^{H}$, where $\mathbf{D}=558$ $\operatorname{diag}\left(s_{1} \mathbf{A}_{1}, s_{2} \mathbf{A}_{2}, \ldots, s_{h} \mathbf{A}_{h}\right)$. Since $\mathbf{N}^{\prime k}=\left(\mathbf{N} \otimes \mathbf{I}_{g}\right)^{k}=\mathbf{N}^{k} \otimes \mathbf{I}_{g}, 559$ we have

$$
\begin{aligned}
\mathbf{N}^{\prime k} \mathbf{N}^{\prime k^{H}} & =\left(\mathbf{N}^{k} \otimes \mathbf{I}_{g}\right)\left(\mathbf{N}^{k} \otimes \mathbf{I}_{g}\right)^{H} \\
& =\left(\mathbf{N}^{k} \mathbf{N}^{k} \otimes \mathbf{I}_{g}\right) \\
& =\mathbf{I}_{h} \otimes \mathbf{I}_{g}=\mathbf{I}_{n}
\end{aligned}
$$

Thus, we have

$$
\begin{aligned}
\mathbf{S S}^{H} & =\mathbf{D D}^{H} \\
& =\operatorname{diag}\left(\mathbf{A}_{1} \mathbf{A}_{1}^{H}, \mathbf{A}_{2} \mathbf{A}_{2}^{H}, \ldots, \mathbf{A}_{h} \mathbf{A}_{h}^{H}\right) \\
& =\operatorname{diag}\left(\mathbf{I}_{g}, \mathbf{I}_{g}, \ldots, \mathbf{I}_{g}\right)=\mathbf{I}_{n} .
\end{aligned}
$$

This concludes the proof. 


\section{REFERENCES}

1] I. E. Telatar, "Capacity of multi-antenna Gaussian channels," Eur. Trans. Telecommun., vol. 10, no. 6, pp. 585-595, Dec. 1999.

2] P. Wolniansky, G. Foschini, G. Golden, and R. Valenzuela, "V-BLAST: An architecture for realizing very high data rates over the rich-scattering wireless channel," in Proc. Int. Symp. Signals, Syst., Electron., Pisa, Italy, Sep. 1998, pp. 295-300.

3] D. Tse and P. Viswanath, Fundamentals of Wireless Communication. Cambridge, U.K.: Cambridge Univ. Press, 2005.

4] B. Hassibi and B. M. Hochwald, "How much training is needed in multiple-antenna wireless links?" IEEE Trans. Inf. Theory, vol. 49, no. 4, pp. 951-963, Apr. 2003.

5] R. Mesleh, H. Haas, C. Ahn, and S. Yun, "Spatial modulation-A new low complexity spectral efficiency enhancing technique," in Proc. 1st Int. Conf. Commun. Netw., Beijing, China, Oct. 2006, pp. 1-5.

[6] R. Mesleh, H. Haas, S. Sinanovic, C. Ahn, and S. Yun, "Spatial modulation," IEEE Trans. Veh. Technol., vol. 57, no. 4, pp. 2228-2242, Jul. 2008.

7] M. Di Renzo, H. Haas, and P. M. Grant, "Spatial modulation for multipleantenna wireless systems-A survey," IEEE Commun. Mag., vol. 49, no. 12, pp. 182-191, Dec. 2011.

8] M. Di Renzo, H. Haas, A. Ghrayeb, S. Sugiura, and L. Hanzo, "Spatial modulation for generalized MIMO: Challenges, opportunities, and implementation," Proc. IEEE, vol. 102, no. 1, pp. 56-103, Jan. 2014

[9] E. Basar, U. Aygolu, E. Panayirci, and H. V. Poor, "Space-time block coding for spatial modulation," IEEE Trans. Commun., vol. 59, no. 3, pp. 823-832, Mar. 2011.

0] M. Di Renzo and H. Haas, "Transmit-diversity for spatial modulation (SM): Towards the design of high-rate spatially-modulated space-time block codes," in Proc. IEEE Int. Conf. Commun., Jun. 2011, pp. 1-6.

11] R. Rajashekar and K. V. S. Hari, "Modulation diversity for spatial modulation using complex interleaved orthogonal design," in Proc. IEEE TENCON, Nov. 2012, pp. 1-6.

2] X. Li and L. Wang, "High rate space-time block coded spatial modulation with cyclic structure," IEEE Commun. Lett., vol. 18, no. 4, pp. 532-535, Apr. 2014

13] J. Jeganathan, A. Ghrayeb, and L. Szczecinski, "Spatial modulation: Optimal detection and performance analysis," IEEE Commun. Lett., vol. 12, no. 8, pp. 545-547, Aug. 2008.

14] S. Sugiura, C. Xu, S. X. Ng, and L. Hanzo, "Reduced-complexity coherent versus non-coherent QAM-aided space-time shift keying," IEEE Trans. Commun., vol. 59, no. 11, pp. 3090-3101, Nov. 2011.

5] J. Wang, S. Jia, and J. Song, "Signal vector based detection scheme for spatial modulation," IEEE Commun. Lett., vol. 16, no. 1, pp. 19-21, Jan. 2012.

16] Q. Tang, Y. Xiao, P. Yang, Q. Yu, and S. Li, “A new low-complexity nearML detection algorithm for spatial modulation," IEEE Wireless Commun. Lett., vol. 2, no. 1, pp. 90-93, Feb. 2013.

7] R. Rajashekar, K. V. S. Hari, and L. Hanzo, "Reduced-complexity ML detection and capacity-optimized training for spatial modulation systems," IEEE Trans. Commun., vol. 62, no. 1, pp. 112-125, Jan. 2014

18] P. Yang, Y. Xiao, Y. Yu, and S. Li, "Adaptive spatial modulation for wireless MIMO transmission systems," IEEE Commun. Lett., vol. 15, no. 6, pp. 602-604, Jun. 2011.

19] P. Yang et al., "Link adaptation for spatial modulation with limited feedback," IEEE Trans. Veh. Technol., vol. 61, no. 8, pp. 3808-3813, Oct. 2012.

20] R. Rajashekar, K. V. S. Hari, and L. Hanzo, "Antenna selection in spatial modulation systems," IEEE Commun. Lett., vol. 17, no. 3, pp. 521-524, Mar. 2013.

21] R. Rajashekar, K. V. S. Hari, and L. Hanzo, "Quantifying the transmit diversity order of Euclidean distance based antenna selection in spatial modulation," IEEE Signal Procss. Lett., vol. 22, no. 9, pp. 1434-1437, Sep. 2015.

22] J. Zheng and J. Chen, "Further complexity reduction for antenna selection in spatial modulation systems," IEEE Commun. Lett., vol. 19, no. 6, pp. 937-940, Jun. 2015.

23] E. Basar, U. Aygolu, E. Panayirci, and H. V. Poor, "Performance of spatial modulation in the presence of channel estimation errors," IEEE Commun. Lett., vol. 16, no. 2, pp. 176-179, Feb. 2012.

24] S. Sugiura and L. Hanzo, "Effects of channel estimation on spatial modulation," IEEE Signal Process. Lett., vol. 19, no. 12, pp. 805-808, Dec. 2012.

25] R. Mesleh, O. S. Badarneh, A. Younis, and H. Haas, "Performance analysis of spatial modulation and space-shift keying with imperfect channel estimation over generalized $\eta-\mu$ fading channels," IEEE Trans. Veh. Technol., vol. 64, no. 1, pp. 88-96, Jan. 2015.
[26] Y. Bian et al., "Differential spatial modulation," IEEE Trans. Veh. 639 Technol., vol. 64, no. 7, pp. 3262-3268, Jul. 2015.

[27] N. Ishikawa and S. Sugiura, "Unified differential spatial modulation," 641 IEEE Wireless Commun. Lett., vol. 3, no. 4, pp. 337-340, Aug. 2014.

[28] W. Zhang, Q. Yin, and H. Deng, "Differential full diversity spatial mod- 643 ulation and its performance analysis with two transmit antennas," IEEE 644 Commun. Lett., vol. 19, no. 4, pp. 677-680, Apr. 2015.

645

[29] M. Wen, X. Cheng, Y. Bian, and H. V. Poor, "A low-complexity near-ML 646 differential spatial modulation detector," IEEE Signal Process. Lett., 647 vol. 22, no. 11, pp. 1834-1838, Nov. 2015.

[30] N. Jacobson, Basic Algebra I, 2nd ed. New York, NY, USA: Wiley, 649 1985.

[31] B. L. Hughes, "Differential space-time modulation," IEEE Trans. Inf. 651 Theory, vol. 46, no. 7, pp. 2567-2578, Nov. 2000.

652

[32] B. M. Hochwald and W. Sweldens, "Differential unitary space-time 653 modulation," IEEE Trans. Commun., vol. 48, no. 12, pp. 2041-2052, 654 Dec. 2000.

[33] B. A. Sethuraman and B. Sundar Rajan, "Full-diversity, high-rate 656 space-time block codes from division algebras," IEEE Trans. Inf. Theory, 657 vol. 49, no. 10, pp. 2596-2616, Oct. 2003.

[34] H. Men and M. Jin, "A low-complexity ML detection algorithm for spatial 659 modulation systems with M PSK constellation," IEEE Commun. Lett., 660 vol. 18, no. 8, pp. 1375-1378, Aug. 2014.

[35] J. Wang, S. Jia, and J. Song, "Generalised spatial modulation system 662 with multiple active transmit antennas and low complexity detection 663 scheme," IEEE Trans. Wireless Commun., vol. 11, no. 4, pp. 1605-1615, 664 Apr. 2012.

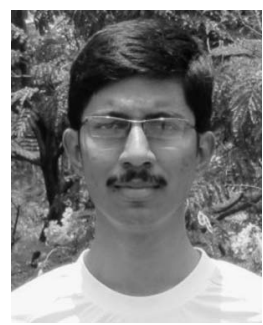

Rakshith Rajashekar (M'15) received the B.E. de- 666 gree in electrical communication engineering from 667 Visvesvaraya Technological University, Belgaum, 668 India, in 2007 and the Ph.D. degree from the 669 Department of Electrical Communication Engineer- 670 ing, Indian Institute of Science, Bangalore, India, 671 in 2014.

672

He is currently a Research Fellow with the Uni- 673 versity of Southampton (UoS), Southampton, U.K. 674 Before joining the UoS, he was a Systems Engineer 675 with Accord Software and Systems, Bangalore, from 676 2007 to 2009 and a Senior Scientist with Broadcom Communications from 677 2014 to 2015. His current research interests include millimeter-wave commu- 678 nication and visible light communication, with a focus on space-time signal 679 processing and coding aspects.

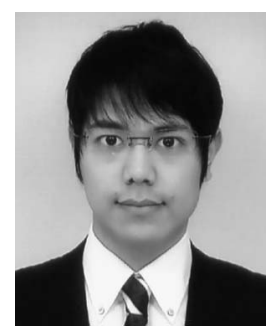

Japanese Government.
Naoki Ishikawa (S'13) was born in Kanagawa, 681 Japan, in 1991. He received the B.E. and M.E. 682 degrees (with highest distinction) in computer and 683 information sciences from the Tokyo University of 684 Agriculture and Technology, Koganei, Japan, in 2014685 and 2015, respectively, where he is currently working 686 toward the Ph.D. degree. 687

From June 2015 to September 2015, he was 688 an academic visitor with the School of Electronics 689 and Computer Science, University of Southampton, 690 Southampton, U.K., which is fully funded by the 691

Mr. Ishikawa has received six domestic student awards, including the 693 Telecom System Technology Student Award (honorable mention) from the 694 Telecommunications Advancement Foundation of Japan in 2014, the Outstand- 695 ing Paper Award for Young C\&C Researchers from the NEC Computer and 696 Communications Foundation in 2014, the Young Researcher's Encouragement 697 Award from the IEEE Vehicular Technology Society Japan Chapter in 2014, 698 and three honors from the Tokyo University of Agriculture and Technology. 699 


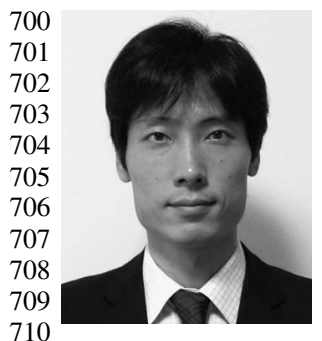

Shinya Sugiura (M'06-SM'12) received the B.S. and M.S. degrees in aeronautics and astronautics from Kyoto University, Kyoto, Japan, in 2002 and 2004, respectively, and the Ph.D. degree in electronics and electrical engineering from the University of Southampton, Southampton, U.K., in 2010.

From 2004 to 2012, he was a Research Scientist with Toyota Central Research and Development Laboratories, Inc., Aichi, Japan. Since 2013, he has been an Associate Professor with the Department of Computer and Information Sciences, Tokyo Uni711 versity of Agriculture and Technology, Koganei, Japan, where he heads the 712 Wireless Communications Research Group. He has authored or coauthored 713 over 70 refereed research publications, including 41 IEEE journal and magazine 714 papers. His research has covered a range of areas in wireless communications, 715 networking, signal processing, and antenna technology.

716 Dr. Sugiura has received a number of awards, including the 14th Funai In717 formation Technology Award (First Prize) from the Funai Foundation in 2015, 718 the 28th Telecom System Technology Award from the Telecommunications 719 Advancement Foundation in 2013, the Sixth IEEE Communications Society 720 Asia-Pacific Outstanding Young Researcher Award in 2011, the 13th Ericsson 721 Young Scientist Award in 2011, and the 2008 IEEE Antennas and Propagation 722 Society Japan Chapter Young Engineer Award. He was also certified as an 723 Exemplary Reviewer for the IEEE COMMUNICATIONS LETTERS in 2013 and 7242014.

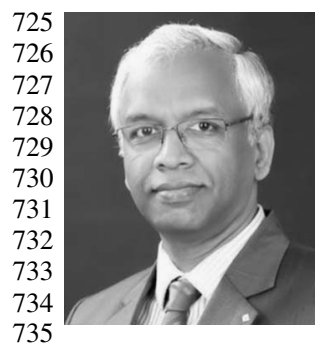

K. V. S. Hari (M'92-SM'97-F'15) received the B.E degree from Osmania University, Hyderabad, India, in 1983; the M.Tech. degree from the Indian Institute of Technology Delhi, New Delhi, India, in 1985; and the $\mathrm{Ph} . \mathrm{D}$. degree from the University of California at San Diego, La Jolla, CA, USA, in 1990.

Since 1992, he has been with the Department of Electrical Communication Engineering, Indian Institute of Science, Bangalore, India, where he is currently a Professor and coordinates the activities of the Statistical Signal Processing Laboratory. He is also 736 an Affiliated Professor with the Department of Signal Processing, KTH Royal 737 Institute of Technology, Stockholm, Sweden. He has been a Visiting Faculty 738 Member with Stanford University, Stanford, CA, USA; KTH Royal Institute 739 of Technology, Stockholm, Sweden; and Aalto University, Espoo, Finland 740 (formerly Helsinki University of Technology). While at Stanford University, he 741 worked on multiple-input multiple-output (MIMO) wireless channel modeling 742 and coauthored the Worldwide Interoperability for Microwave Access stan743 dard on wireless channel models for fixed-broadband wireless communication 744 systems, which proposed the Stanford University Interim channel models. $745 \mathrm{He}$ was also with the Defense Electronics Research Laboratory, Hyderabad, 746 and the Research and Training Unit for Navigational Electronics, Osmania 747 University. His research interests include the development of signal processing 748 algorithms for MIMO wireless communication systems, sparse signal recovery 749 problems, indoor positioning, assistive technologies for the elderly, and visual 750 neuroscience.

751 Dr. Hari is currently an Editor of Elsevier's EURASIP journal Signal 752 Processing and the Senior Associate Editor of Springer's Indian Academy of 753 Sciences journal SADHANA. He received the Institution of Electronics and 754 Telecommunication Engineers S. V. C. Aiya Award for Excellence in Telecom 755 Education and the Distinguished Alumnus Award from the Osmania University 756 College of Engineering, Hyderabad. He is an Academic Entrepreneur and a 757 Cofounder of the company ESQUBE Communication Solutions, Bangalore.

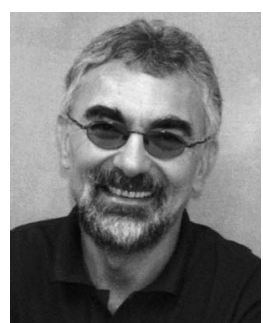

Lajos Hanzo (M'91-SM'92-F'04) received the 758 M.S. degree in electronics and the Ph.D. degree from 759 Budapest University of Technology and Economics 760 (formerly, Technical University of Budapest), 761 Budapest, Hungary, in 1976 and 1983, respec- 762 tively; the D.Sc. degree from the University of 763 Southampton, Southampton, U.K., in 2004; and 764 the "Doctor Honoris Causa" degree from Budapest 765 University of Technology and Economics in 2009. 766

During his 38-year career in telecommunications, 767 he has held various research and academic posts in 768 Hungary, Germany, and the U.K. Since 1986, he has been with the School 769 of Electronics and Computer Science, University of Southampton, where he 770 holds the Chair in Telecommunications. He is currently directing a 100-strong 771 academic research team, working on a range of research projects in the 772 field of wireless multimedia communications sponsored by the industry, the 773 Engineering and Physical Sciences Research Council, the European IST Pro- 774 gram, and the Mobile Virtual Center of Excellence. During 2008-2012, he 775 was a Chaired Professor with Tsinghua University, Beijing, China. He is an 776 enthusiastic supporter of industrial and academic liaison and offers a range of 777 industrial courses. He has successfully supervised more than $80 \mathrm{Ph}$.D. students, 778 coauthored 20 John Wiley/IEEE Press books on mobile radio communications 779 totaling in excess of 10000 pages, and published more than 1300 research 780 entries at IEEE Xplore. He has more than 17000 citations. His research is 781 funded by the European Research Council's Senior Research Fellow Grant. 782 (For further information on research in progress and associated publications, 783 please refer to http://www-mobile.ecs.soton.ac.uk.)

Dr. Hanzo is a Fellow of the Royal Academy of Engineering, The Institution 785 of Engineering and Technology, and the European Association for Signal 786 Processing. He is also a Governor of the IEEE Vehicular Technology Society. 787 He has served as the Technical Program Committee Chair and the General Chair 788 of IEEE conferences, has presented keynote lectures, and has been awarded a 789 number of distinctions. During 2008-2012, he was the Editor-in-Chief of the 790 IEEE Press. 


\section{AUTHOR QUERY}

NO QUERY. 